\title{
Numerical Study of the Generic Sports Utility Vehicle Design with a Drag Reduction Add-On Device
}

\author{
Shubham Singh, M. Zunaid, Naushad Ahmad Ansari, Shikha Bahirani, \\ Sumit Dhall, and Sandeep Kumar \\ Department of Mechanical Engineering, Delhi Technological University, Bawana Road, Shahbad Daulatpur, Delhi 110042, India \\ Correspondence should be addressed to Shubham Singh; shubham.singh091@gmail.com
}

Received 28 July 2014; Revised 21 October 2014; Accepted 11 November 2014; Published 17 December 2014

Academic Editor: Fu-Yun Zhao

Copyright (c) 2014 Shubham Singh et al. This is an open access article distributed under the Creative Commons Attribution License, which permits unrestricted use, distribution, and reproduction in any medium, provided the original work is properly cited.

\begin{abstract}
CFD simulations using ANSYS FLUENT 6.3.26 have been performed on a generic SUV design and the settings are validated using the experimental results investigated by Khalighi. Moreover, an add-on inspired by the concept presented by Englar at GTRI for drag reduction has been designed and added to the generic SUV design. CFD results of add-on model and the basic SUV model have been compared for a number of aerodynamic parameters. Also drag coefficient, drag force, mean surface pressure, mean velocities, and Cp values at different locations in the wake have been compared for both models. The main objective of the study is to present a new add-on device which may be used on SUVs for increasing the fuel efficiency of the vehicle. Mean pressure results show an increase in the total base pressure on the SUV after using the device. An overall reduction of $8 \%$ in the aerodynamic drag coefficient on the add-on SUV has been investigated analytically in this study.
\end{abstract}

\section{Introduction}

Sports utility vehicles are known worldwide for their ruggedness and high passenger capacity. Their ability to run on both off-road and on-road conditions makes them very peculiar for usage. Due to their high utility, a need for making them more fuel efficient has arisen in the past few years. The following data shows the average miles per gallon statistics for cars and SUVs. The increase in the fuel efficiency is due to improvements in the aerodynamics of the automobiles. For the SUVs the average miles per gallon has been fairly increasing in the past few years as shown in Figure 1. The need of lowering down the aerodynamic drag on the SUVs in order to further increase the average miles per gallon has been the issue for the future work.

Aerodynamic effects on a running SUV play a substantial role in their fuel efficiency. Efforts have been made for making the SUVs aerodynamically and fuel efficient by using a variety of add-on devices and other methods. Shape cannot be much compromised for the basic purpose of the SUV. Hence methods to alter the air flow over the surface of the SUV by using devices or by blowing air in the rear region of the SUV are widely used for drag reduction. Form drag or the pressure drag contributes to the $90 \%$ of the total drag [1] on the bluff bodies and can be reduced using the add-on devices.

A great amount of research has been done in the field of add-on devices on SUVs and pick-up trucks for reduction in drag forces by altering the air flow around and behind the vehicle. Some of the work has been reported here. Englar Robert [2] at Georgia Tech has reported a drag reduction of $84 \%$ on heavy vehicles using pneumatic devices. van Leeuwen [3] has achieved a drag reduction of $15 \%$ to $20 \%$ over a GETS (generic European transportation system) model. Lamond et al. [4] investigated a base bleed method of drag reduction over a production SUV design and achieved a maximum drag reduction of $10.7 \%$. Sevilla [5] used Vortex generators as a method for reattachment of flow over the generic SUV and reported a maximum drag reduction of $2.7 \%$. Krishnani [6] investigated the effect of varying inclination of the upper and lower boat tail plates on the generic SUV and reported a maximum drag reduction of $12.3 \%$. Wahba et al. [7] used lateral guide vanes of different geometries over a SUV and reported a maximum drag reduction of up to $18 \%$. Bahram et al. [8] experimented with a square back bluff body using tail and boat plates for drag reduction and were able to reduce the drag coefficient by $20 \%$. 


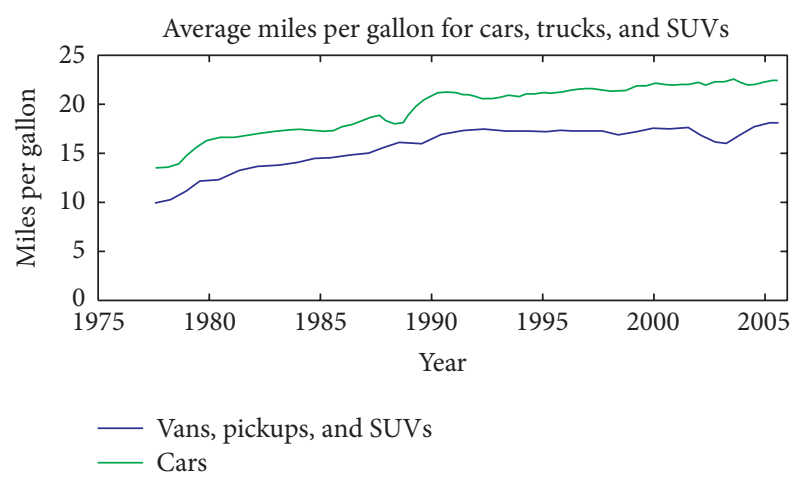

FIGURE 1: Average miles per gallon for automobiles in US from 1970 to 2006 .

Boat and tail add-ons have been used on a large scale over the years. Add-ons with different shapes and sizes have been experimented and substantial results have been worked out. In this study, a boat tail add-on using the concept of Coanda effect [10] has been used for drag reduction. The add-on has been added at the base of a generic SUV design and numerical simulations have been done using commercial package ANSYS FLUENT 6.3.26. Steady state RANS simulations have been made for a Reynolds number of $8.87 * 10^{5}$. The aerodynamic flow has been studied for various parameters and a comprehensive comparison with the basic model of the SUV has been provided.

The main features of the flow being observed using the wake pathlines are a strong downwash from the cab surface of the SUV and a strong upwash from the underbody surface. The same was reported by Al-Garni et al. [9] experimentally. The pathlines from underbody form an anticlockwise vortex in the lower region of the wake and the upwash forms a clockwise rotating vortex in the upper rear region of the wake.

\section{Basic Model Case: Validation of CFD Settings}

The basic model of the generic SUV design has been numerically solved using the RANS kw(sst) model at a Reynolds number of $8.87 * 10^{5}$. Al-Garni et al. [9] at the University of Michigan performed the study on the basic SUV model in a wind tunnel and produced results have been used to validate the case. The flow is numerically solved using the laws of conservation of mass and the conservation of momentum. Energy equation has been omitted during the solution and the compressibility in the flow has been ignored. The basic equations are given below:

conservation of mass:

$$
\begin{gathered}
\nabla \cdot v=0 \\
\frac{\partial \rho}{\partial t}+\nabla \cdot(\rho v)=0
\end{gathered}
$$

conservation of momentum:

$$
\rho \frac{D v}{D t}=-\nabla P+\mu \Delta v
$$

$\mathrm{Kw}$ (sst) was used as the turbulence model to capture the turbulence in the wake and the boundary region. The kw(sst) turbulence equations are given as follows:

kinematic eddy viscosity:

$$
v_{t}=\frac{a_{1} k}{\max \left(a_{1} \omega, \mathrm{SF}_{2}\right)}
$$

turbulence kinetic energy:

$$
\frac{\partial k}{\partial t}+U_{j} \frac{\partial k}{\partial x_{j}}=P_{k}-\beta^{*} k \omega+\frac{\partial}{\partial x_{j}}\left[\left(v+\sigma_{k} v_{T}\right) \frac{\partial k}{\partial x_{j}}\right]
$$

specific dissipation rate:

$$
\begin{aligned}
\frac{\partial \omega}{\partial t}+U_{j} \frac{\partial \omega}{\partial x_{j}}= & \alpha S^{2}-\beta \omega^{2}+\frac{\partial}{\partial x_{j}}\left[\left(v+\sigma_{\omega} v_{T}\right) \frac{\partial \omega}{\partial x_{j}}\right] \\
& +2\left(1-F_{1}\right) \sigma_{\omega^{2}} \frac{1}{\omega} \frac{\partial k}{\partial x_{i}} \frac{\partial \omega}{\partial x_{i}}
\end{aligned}
$$

Al-Garni et al. [9] experimented the generic SUV model in a wind tunnel to have a better understanding of the aerodynamics of the wake of the SUV model. They used particle image velocimetry (PIV) techniques to see the complex recirculation region behind the SUV and POD methods to understand the turbulent structure of the wake. They used a number of parameters for studying of the SUV aerodynamics, including the mean pressure coefficient measurements over the SUV surface, drag forces, velocity components (horizontal, vertical, and lateral) in the wake region, pressure and velocity contours and streamlines, and others. We used the data calculated by them as benchmark for validating our CFD settings and further performed simulations. Of all the parameters, three major parameters are used for validation: (1) coefficient of pressure plots on the underbody and cab surface, (2) velocity streamlines in the centre horizontal plane, and (3) mean velocity profiles of the flow in the symmetry and centre horizontal plane $(z=$ $69.2 \mathrm{~mm}$ ) of the wake of the SUV.

\section{SUV Model}

Figures 2 and 3 show the basic dimensions of the generic SUV model used for the validation case. The length of the SUV model used for CFD is $432 \mathrm{~mm}$, height is $148 \mathrm{~mm}$, and the maximum width is $152 \mathrm{~mm}$. Cross-sectional area for calculation of drag coefficient is $0.010799 \mathrm{~m}^{2}$. The coordinate system used for the study is also shown in Figure 3. $x$-axis points in the direction of the free stream velocity. $y$-axis is in the lateral direction and the $z$-axis in the vertical direction. A point at the geometrical centre of the base of the SUV is marked and is called as the base point. Total pressure and coefficient of pressure at the base point are calculated and compared. The basic model is not detailed for the purpose of reducing the mesh size and computational resources. The computational domain is shown in Figure 4 with the lengths being the standard car lengths. SOLIDWORKS is used to 


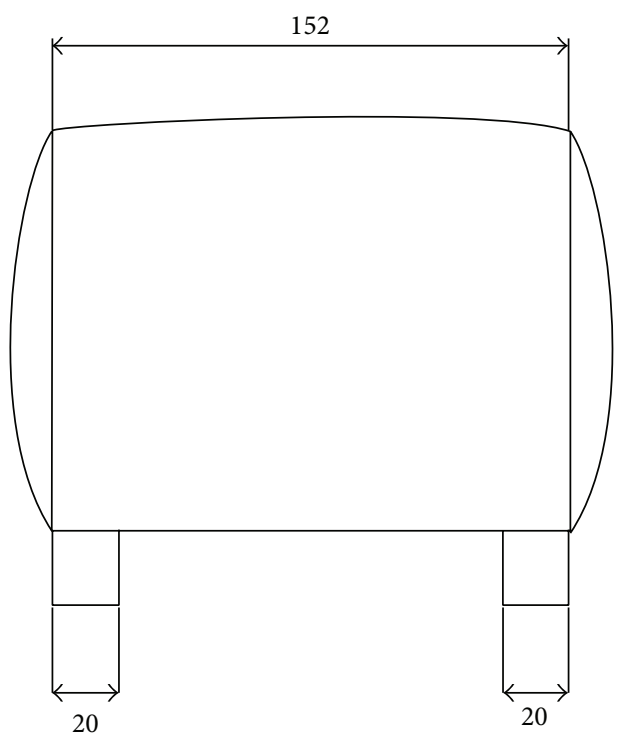

FIGURE 2: Rear view of the basic SUV.

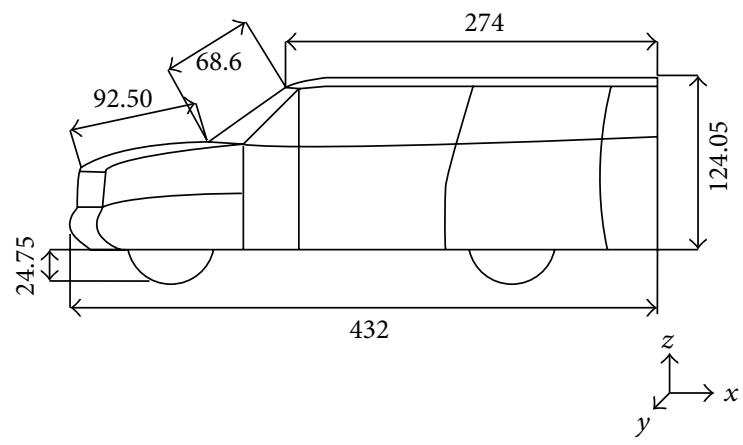

FIgURE 3: Side view of the basic SUV.

build the CAD geometry and GAMBIT 2.4.6 is used to build the mesh, define the computational domain. The dimensions of the computational domain are recommended by FLUENT, Germany [11].

ANSYS FLUENT 6.3.26 is used to simulate the design and finally perform the postprocessing. Mesh included an inflation layer with 5 layers with a growth rate of $20 \%$ surrounding the SUV model to capture the turbulence.

Figure 5 shows the mesh of the half-model which was made using GAMBIT 2.4.6. Mesh method used is the patch conforming tetrahedron with the prismatic elements in the inflation layer. To save the computational resources, only half of the total CAD model was simulated cut by a symmetry plane in the $X Z$ plane. This reduced the mesh size by half of the original and hence the time for the iterations was also reduced. A course mesh with around 1.2 million elements was used for the study. Convergence criteria were set to have the maximum residual error of $10^{-3}$. K-omega(sst) model was used to solve the model as it can be used as low-Re turbulence model. It has a good functionality in adverse pressure gradients and separating flow conditions. The residual parameters used in this context are continuity,

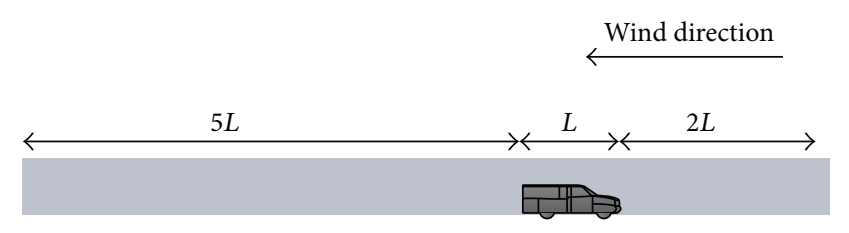

FIGURE 4: Computational domain for the SUV.

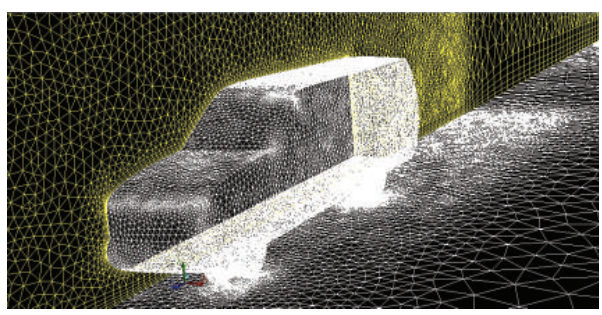

FIGURE 5: Mesh of the basic SUV.

$x$-velocity, $y$-velocity, $z$-velocity, turbulence kinetic energy (k), and specific dissipation rate (omega). The average drag coefficient was calculated and came out to be 0.3569 .

\section{Parameters for Validation}

Postprocessing of the basic model case produced results which were similar to the experimental results by Al-Garni et al. [9]. As explained earlier, the various parameters were compared with the experimental results and similar observations were found.

4.1. Mean Coefficient of Pressure Plots. The mean Cp plots for the basic model for both the cab and the underbody surface were similar to the experimental ones by Al-Garni et al. [9]. Figure 6 shows the plots for the SUV model for both the surfaces of the SUV. All the pressure measurements are done at the free stream velocity of $30 \mathrm{~m} / \mathrm{s}$ and an average Reynolds number of $8.87 * 10^{5}$. In the figure, the dots indicate the pressure values for the cab surface and the arrow heads for the underbody surface.

As stated in Figure 6, the values for mean value of pressure coefficient at the cab surface are similar to the experimental values reported by Al-Garni et al. [9] which helps to validate the CFD settings.

Similarly, for the underbody surface, two local minima can be observed at $x=100$ and $350 \mathrm{~mm}$, where the Cp value is close to -0.3 due to the presence of the front and the rear tires. This was also observed by Al-Garni et al. [9] in their study.

4.2. Velocity Streamlines for SUV Wake in the Centre Horizontal Plane. Figure 7 shows the velocity streamlines for the wake region in the horizontal plane which is found to be similar to the one calculated by Al-Garni et al. [9] experimentally shown in Figure 8. Circulation region extends for a length of around $170 \mathrm{~mm}$ which is 1.1 times the width and the distance between the centers of recirculation is around $100 \mathrm{~mm}$. 


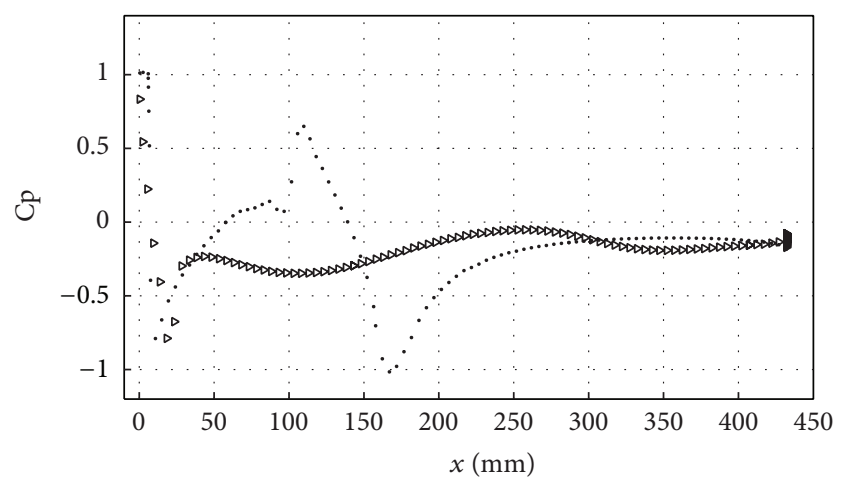

- Cab surface

$\triangle$ Underbody surface

Figure 6: Cp plots for basic SUV.

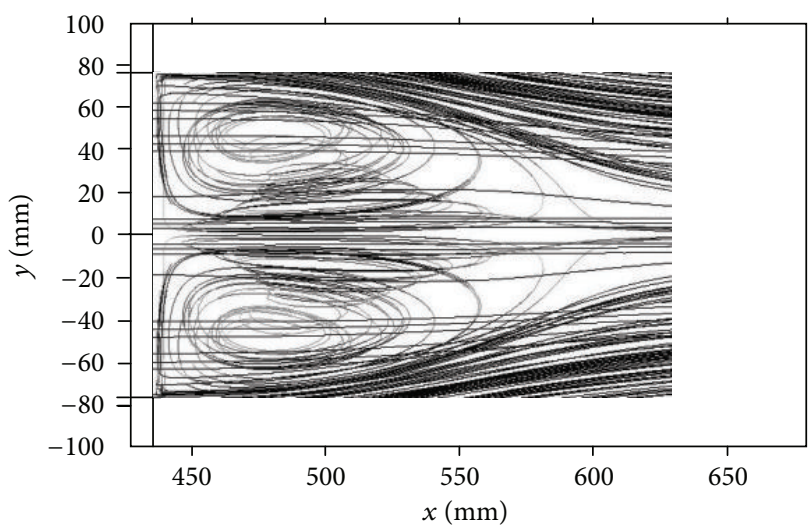

FIGURE 7: Streamlines in the center horizontal plane for basic SUV.

Table 1 compares the values of $\mathrm{Cp}$ between the experimental result by Khalighi and the CFD results on basic SUV.

4.3. Mean Velocity Profiles in the Centre Horizontal Plane of the SUV Wake. Figures 9 and 10 show the mean velocity profiles in the centre horizontal plane at $z=69 \mathrm{~mm}$ for the SUV wake region for both the downstream and the lateral components. The results are identical to one found by Al-Garni et al. [9] in their experimental study. For the downstream component, maximum recirculation velocity is 0.4 times the free stream velocity and the flow reversal occurs between $x=450$ and $600 \mathrm{~mm}$. For the lateral velocity component, the magnitude is maximum at $x=600 \mathrm{~mm}$ at a value of 0.18 times the free stream velocity. For this parameter as well, the results are quite similar to the ones produced by Al-Garni et al. [9].

\section{Add-On Device Used for Drag Reduction}

The add-on device used on the generic SUV is inspired from the GETS (generic European transport system) as mentioned by van Leeuwen [3]. The GETS model has been used earlier for studying the wake of the tractor trailer configuration. The curved boat tail configuration was used with an active flow

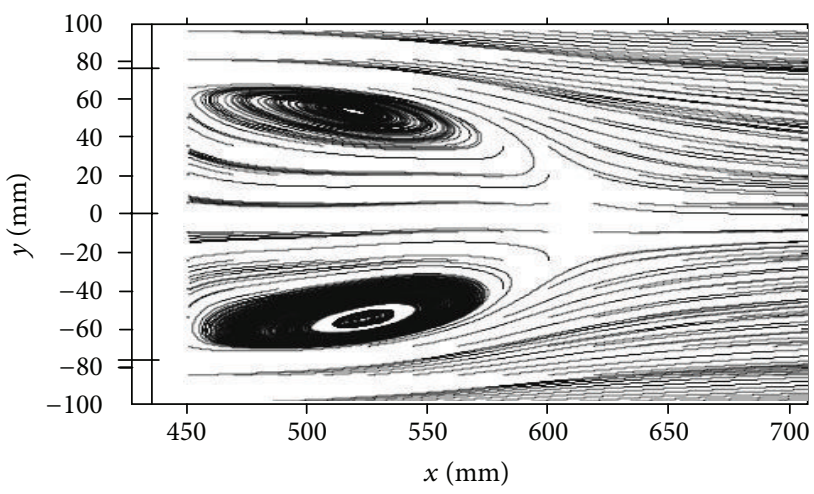

FIGURE 8: Figure of streamlines by Al-Garni et al. [9].

TABLE 1: Comparison of Cp values.

\begin{tabular}{lccc}
\hline S. number $X(\mathrm{~mm})$ & Cp (experimental values) & Cp (CFD values) \\
\hline 1 & 5 & 1.0 & 1.0 \\
2 & 10 & -0.5 & -0.8 \\
3 & 110 & 0.5 & 0.65 \\
4 & 165 & -0.9 & -1.0 \\
5 & 350 & -0.1 & -0.1 \\
\hline
\end{tabular}

control (AFC) device to reduce the drag by repressurizing the wake region [3]. In our case, we have used the curved boat tail configuration without the AFC device on the SUV.

The add-on works on the principle of Coanda effect patented by Coanda [10] in which a flow follows a curved nearby surface, if the curvature is not too sharp. The geometry of the curved boat tail configuration is shown in Figure 11. The design is inspired by the one proposed by van Leeuwen [3]. The add-on is extended fully on the edge of the SUV base. The radius of the curved region is given as 0.115 times the width of the SUV base [3]. Consider the following:

(i) $R 1=R 2=0.115 w$,

(ii) $h=0.00154 w$,

where " $w$ " is the width of the vehicle and " $h$ " is the height from where the jet of stream is blown in the wake region. Consider the following:

(i) $\Theta=90 \mathrm{deg}$,

(ii) $V_{\text {jet }}=0$.

These empirical relations, suggested by van Leeuwen [3], are for the present study as follows.

Since the width of the SUV model $w=152 \mathrm{~mm}$, therefore

(i) $R 1=R 2=17.48 \mathrm{~mm}$,

(ii) $h=0.23408 \mathrm{~mm}$.

Due to the Coanda effect, the air flow over and under the SUV takes a curved path leading to blowing of the air in the immediate wake region-also known as "energizing the dead water" [12]. This leads to increase in the average pressure in 

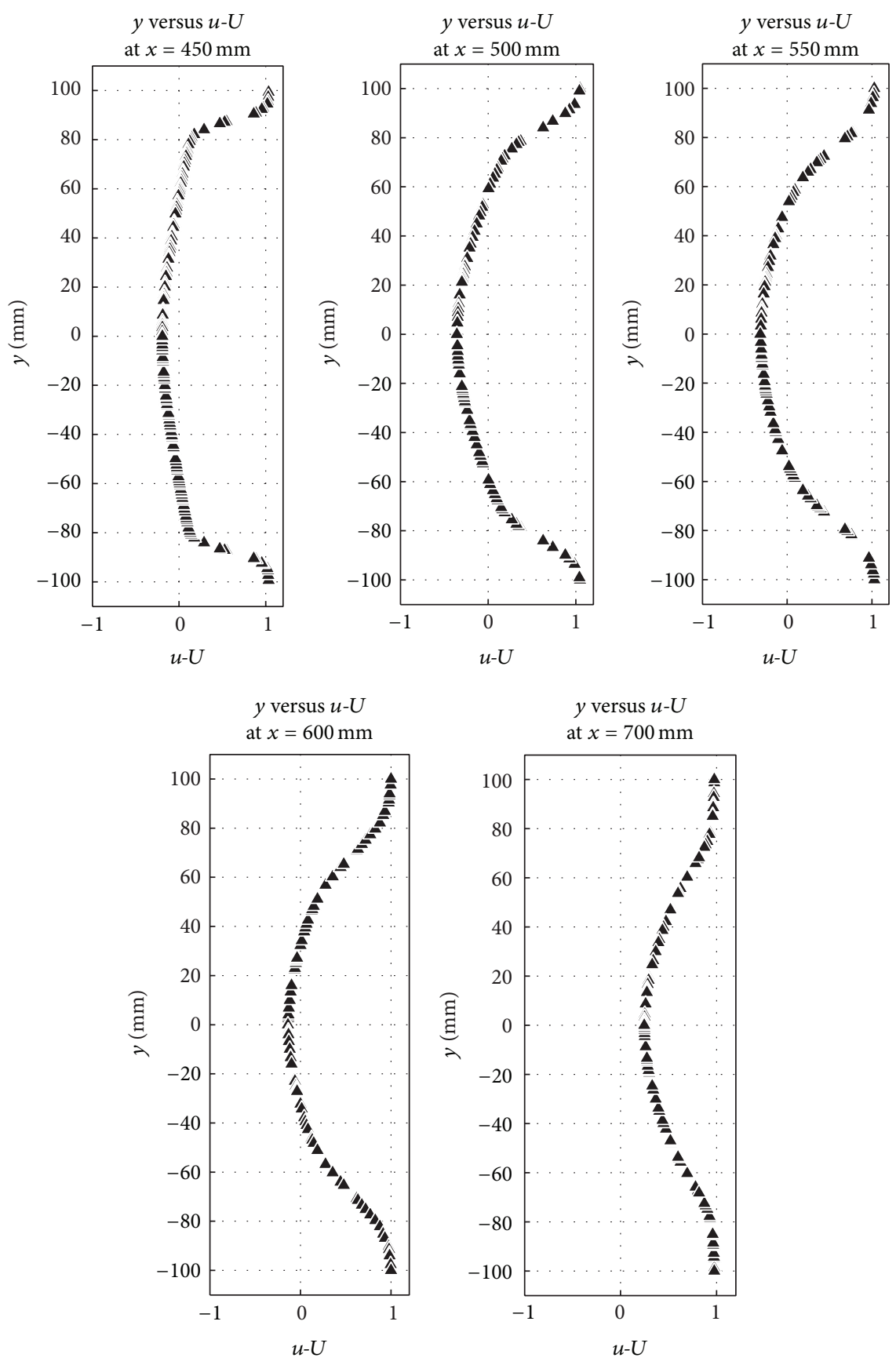

FIGURE 9: Mean velocity profiles for basic SUV, $y$ versus $u$ - $U$.

the wake region and thus reduction in the drag force on the SUV. Figure 12 shows the add-on SUV with curved boat tail configuration installed at the base.

\section{Add-On Model Case}

The add-on SUV model is numerically solved using the ANSYS FLUENT 6.3.26 for a Reynolds number of $8.87 * 10^{5}$ using the RANS kw(sst) model.

Mesh settings used are identical to the basic SUV case. Mesh has been optimized to have 1.2 million elements.
Reference area used for the calculation of the coefficient of drag is $0.01080399 \mathrm{~m}^{2}$. Convergence criteria are set to have a residual error of $10^{-3}$ and the solution was converged in 387 iterations. Similar to the basic SUV case, an inflation layer with 5 layers and a growth rate of $20 \%$ was used on the cab and the underbody boundary. Rear and side views of the model are shown in Figures 13 and 14, respectively, with all the dimensions. A similar base point is also marked in the add-on SUV for calculation of the average pressure and coefficient of pressure at the base of the SUV. Similar to the basic model case, $\mathrm{kw}(\mathrm{sst})$ model was used for the 

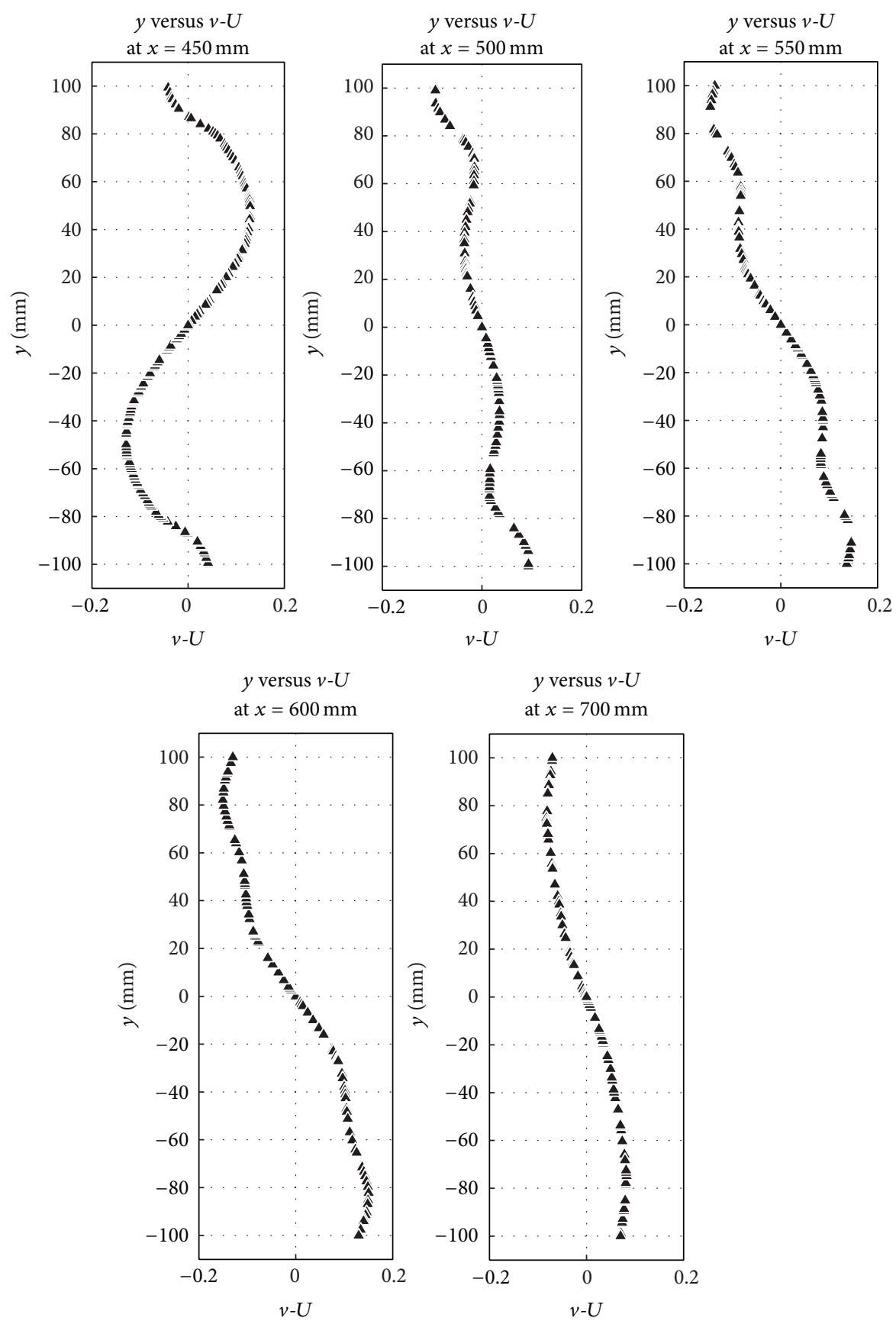

FIgURE 10: Mean velocity profiles for basic SUV, $y$ versus $v$ - $U$.

add-on model case as well. Computational domain used was very similar to the one used for the basic model case. A wide range of aerodynamic parameters are calculated for the addon model case and compared with the basic model case to establish the effectiveness of the curved boat tail add-on used on the generic SUV.

\section{Results and Comparison}

7.1. Average Coefficient of Pressure. The average coefficient of pressure plots have been calculated on the cab and the underbody surface and are compared in Figures 15 and 16, respectively.

For the cab surface case, most of the portion of the curve overlaps with the basic case. However, only noticeable difference is at the base of the SUV. Due to the suction created at the base, the air rushes past the add-on leading to movement of air over a convex portion downwards which causes the velocity to increase and pressure to reduce. However, due to the add-on effect the wake region is repressurized and the average value of $\mathrm{Cp}$ is high as compared to the basic model as shown in the figure. 


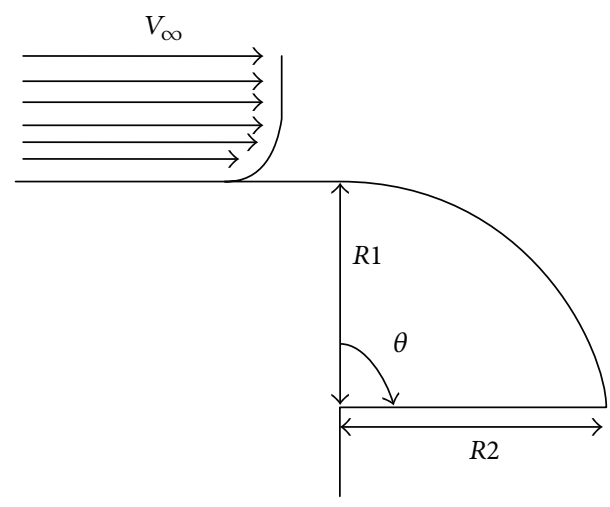

FIGURE 11: Add-on device geometry used on the SUV.

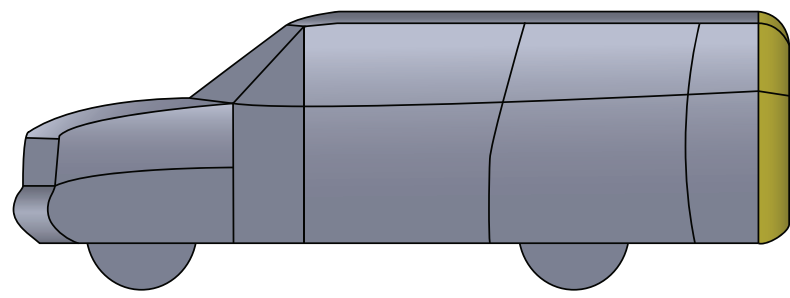

FIGURE 12: SUV with add-on on the rear.

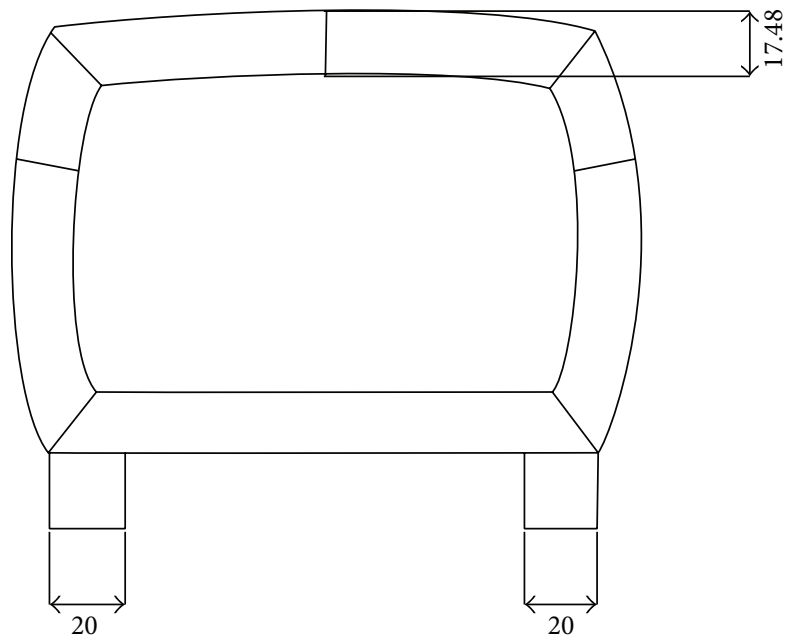

FIGURE 13: Add-on SUV rear view.

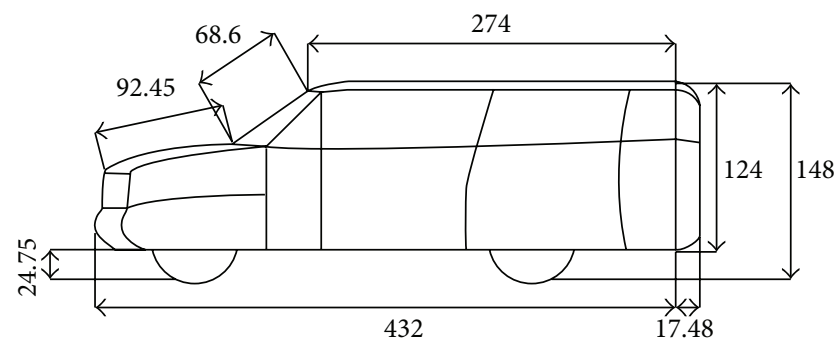

FIGURE 14: Add-on SUV side view.

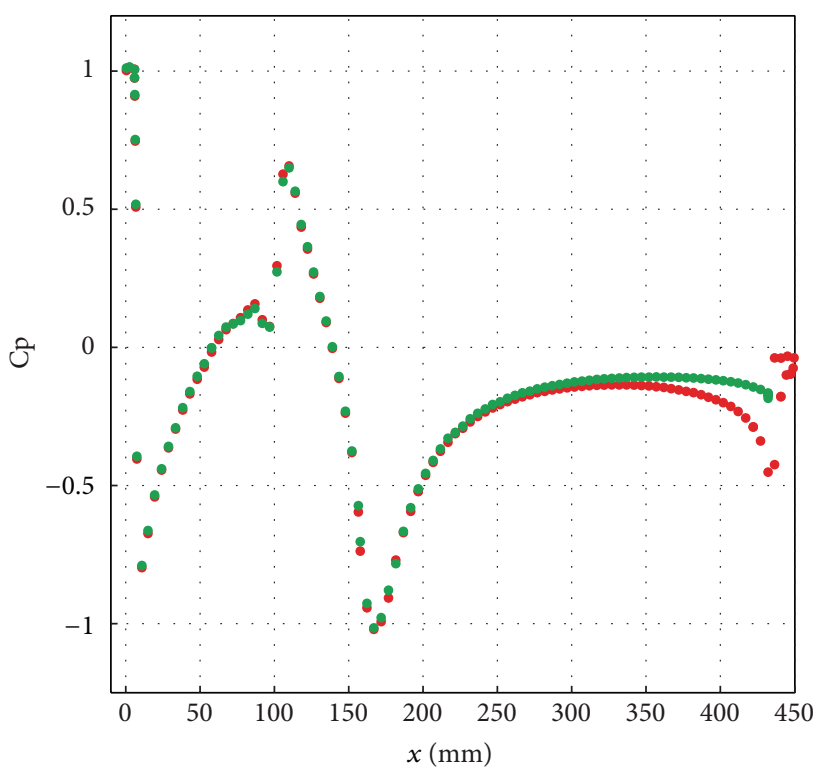

- Basic model

- Add-on model

FIGURE 15: Cp plots comparison for cab surface.

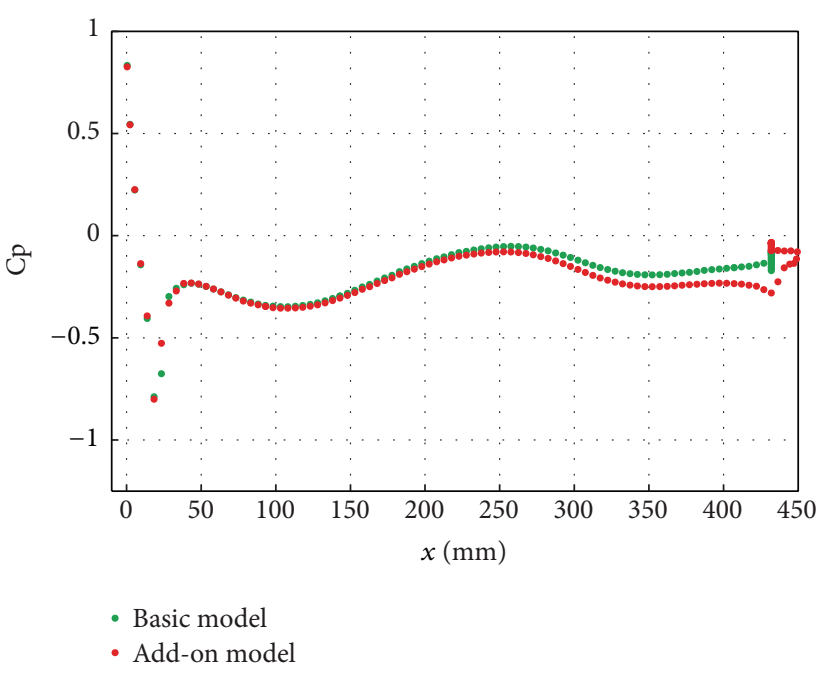

FIgURE 16: Cp plots comparison for underbody surface.

Similar phenomenon occurs for the underbody surface and the average value of $\mathrm{Cp}$ is on the higher side as compared to the basic case leading to reduction in the drag force on the SUV.

7.2. Horizontal Plane. Velocity pathlines and pressure contours have been plotted in the horizontal plane $(z=69.2 \mathrm{~mm})$ for analyzing the flow structure and the pressure values in the wake region.

Figures 17 and 18 show the velocity vectors in the wake region for the add-on and the basic model, respectively. The centers of the recirculation vortices for the basic case are located at $y=+55 \mathrm{~mm}$ and $-55 \mathrm{~mm}$. For the add-on case, the 


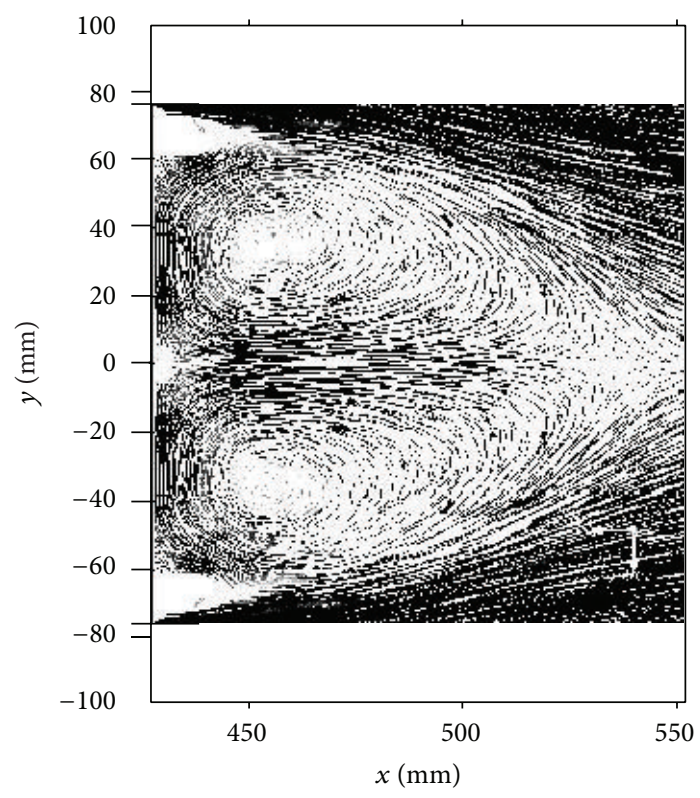

FIGURE 17: Velocity vectors for add-on model SUV.

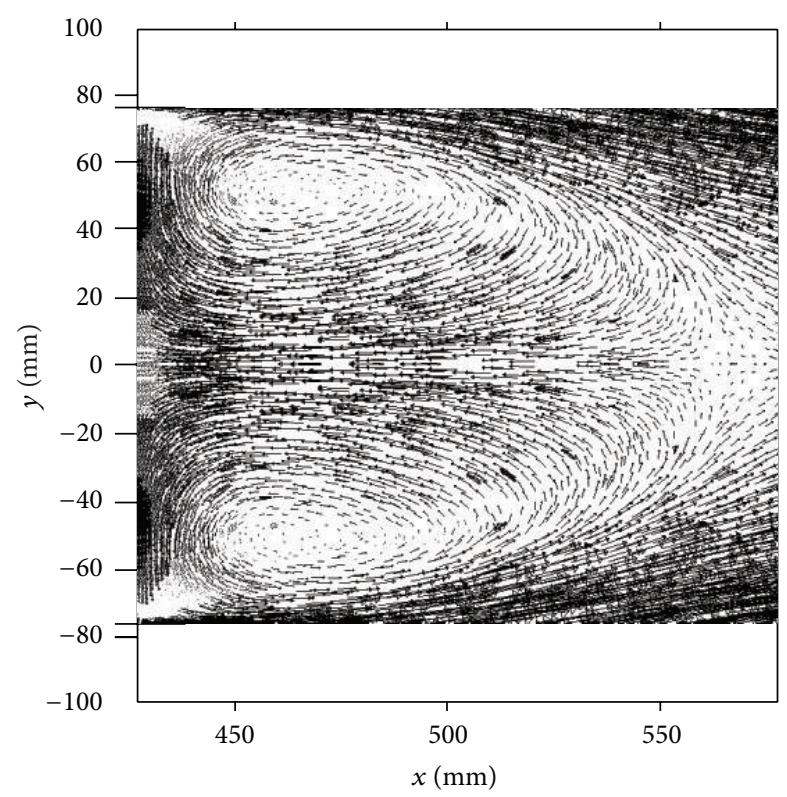

FIGURE 18: Velocity vectors for basic model SUV.

centers are close to each other and are located at $y=+35 \mathrm{~mm}$ and $y=-35 \mathrm{~mm}$. Reduction in the width of the wake region results in the increase in the wake pressure and, therefore, lesser drag on the SUV. Also along the $x$-axis, the location of the recirculation vortices has moved from approximately $475 \mathrm{~mm}$ for the basic model to around $460 \mathrm{~mm}$ for the addon model. This makes the wake shorter for the add-on model as compared to the basic model.

Velocity pathlines in the horizontal plane are shown in Figures 19 and 20 for both the add-on and the basic models, respectively. It is clearly visible that the reattachment region for the pathlines from the cab and the underbody surface for

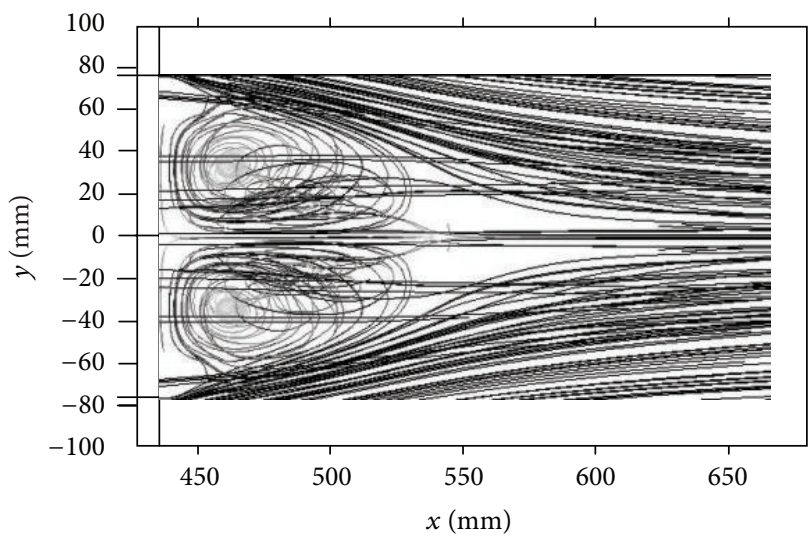

FIGURE 19: Velocity pathlines for add-on model SUV.

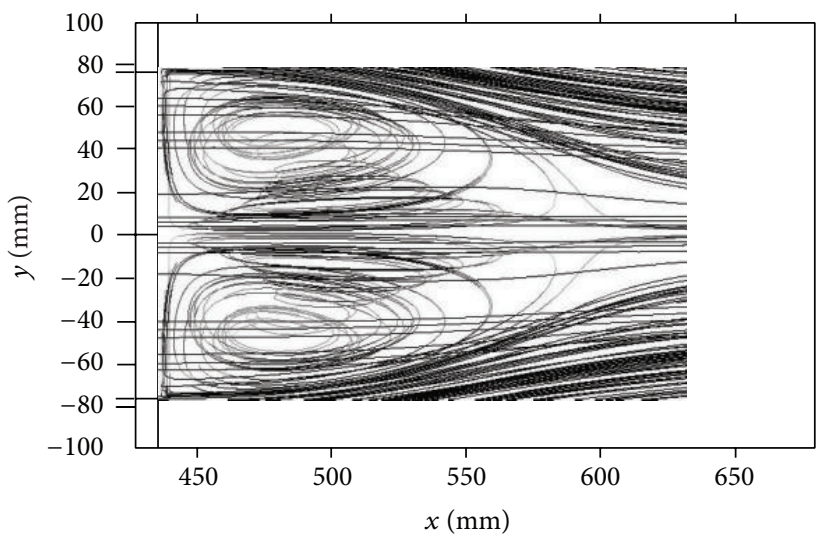

FIGURE 20: Velocity pathlines for basic model SUV.

the basic model lie at around $x=630 \mathrm{~mm}$. For the add-on model, reattachment region lies close to $x=600 \mathrm{~mm}$. Shorter wake region shows higher pressure in the wake region and, hence, lesser drag on the SUV.

Velocity contours for the wake region are shown in Figures 21 and 22 for both the add-on and the basic models, respectively. At $x=500$ for the basic model, the width of wake is approximately $140 \mathrm{~mm}$. For the add-on model, width of the wake at $x=500 \mathrm{~mm}$ is around $120 \mathrm{~mm}$. This proves the reduction in the width of the wake and, hence, ultimately higher pressure in the wake region. Total pressure in the horizontal plane for the add-on and the basic models is shown in the Figures 23 and 24, respectively. The area of low pressure in the ring vortices is more concentrated in case of the addon model as compared to the basic model. The width and the length of the vortices are reduced leading to a high pressure in the ring vortices and hence a higher pressure in the wake.

At $x=450 \mathrm{~mm}$ and $y=40 \mathrm{~mm}$, total pressure is as follows:

(1) for add-on model: $114 \mathrm{~Pa}$;

(2) for basic model: $139 \mathrm{~Pa}$.

(3) Hence, total pressure in the base region is higher for the add-on model as compared to the basic model. 


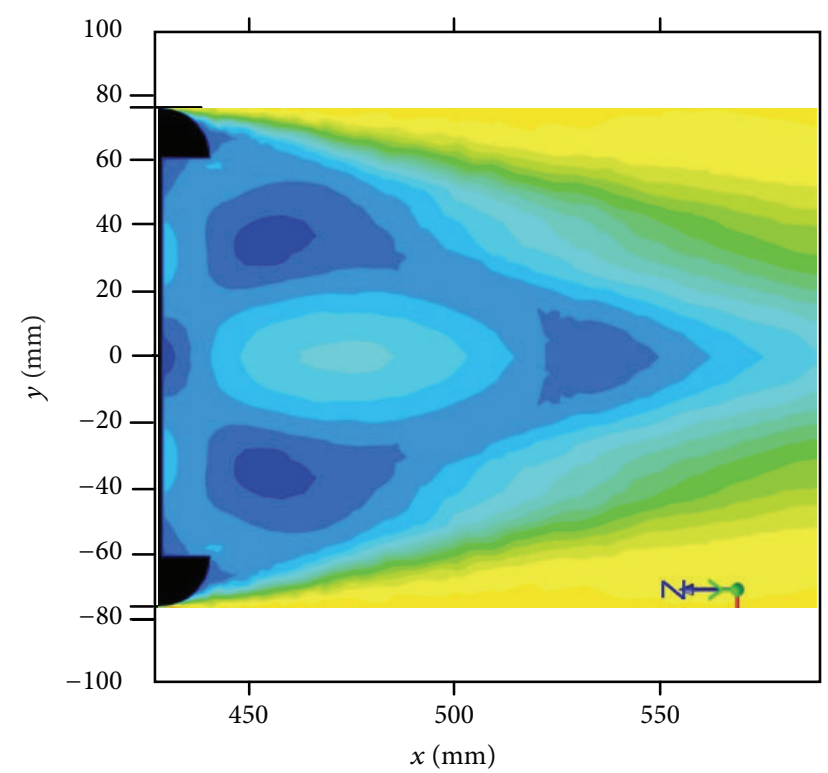

FIGURE 21: Velocity contours in horizontal plane for add-on SUV model.

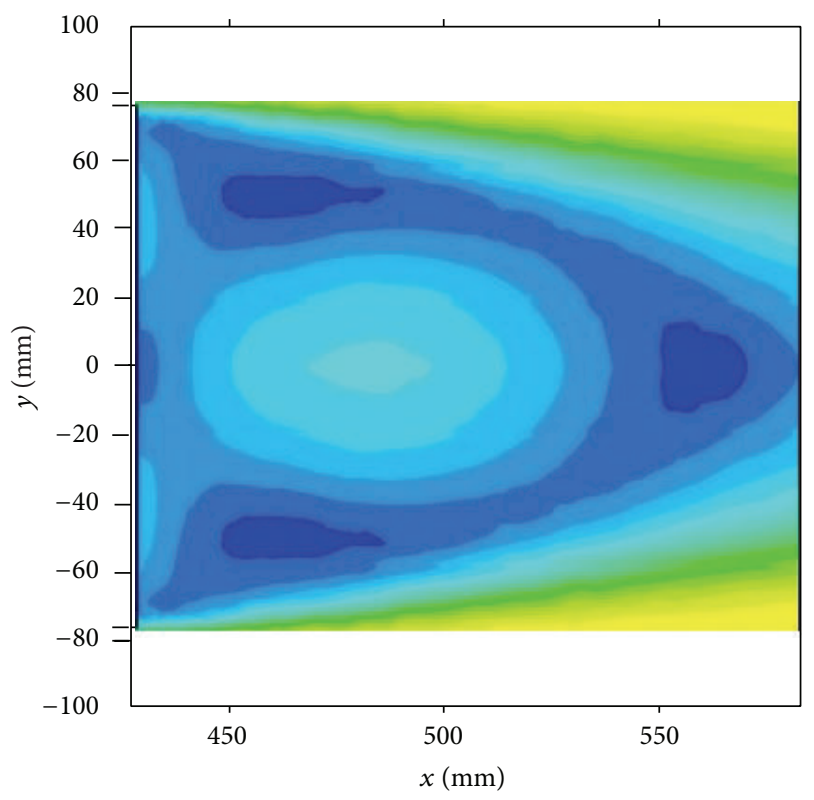

FIGURE 22: Velocity contours in horizontal plane for basic SUV model.

Figure 25 shows the mean velocity values for lateral velocity component in the center horizontal plane for both models. The Coanda effect is clearly seen due to the sudden changes in the velocities for $x=450 \mathrm{~mm}$. Also, a strong side sweep is present for both models throughout the wake region. The value of lateral component of velocities is comparatively higher for the add-on model from $x=500 \mathrm{~mm}$ to $x=700 \mathrm{~mm}$.

The vertical component of velocity in the wake region in the center horizontal plane for both the models is shown in Figure 26. The maximum flow reversal can be observed to be around 0.4 times the free stream velocity for the basic model

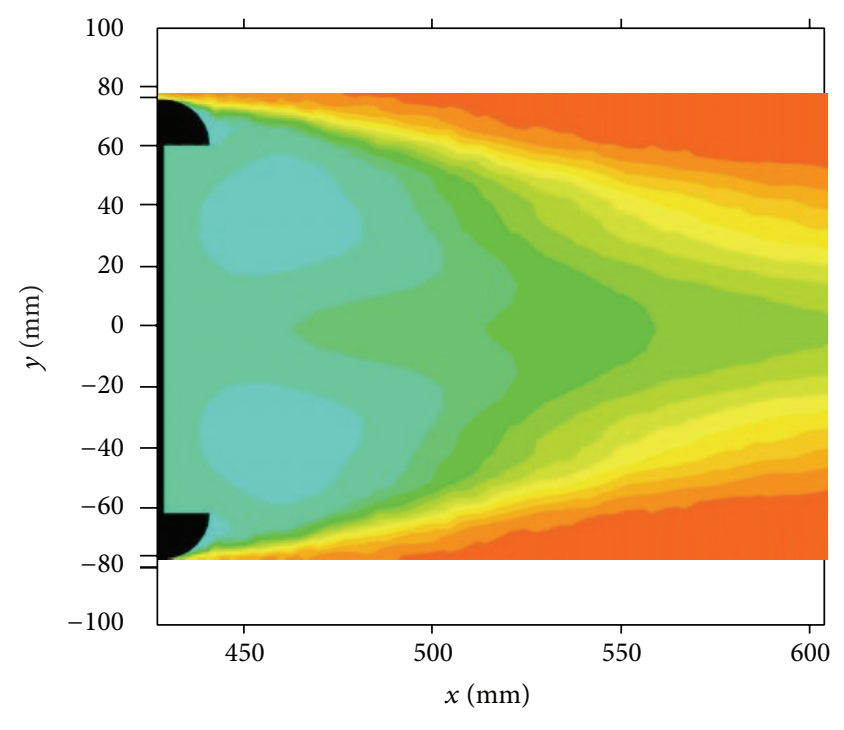

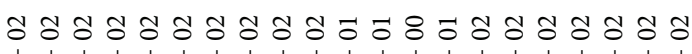
กั้ तु ம்

FIgURE 23: Total pressure in horizontal plane for add-on SUV model.

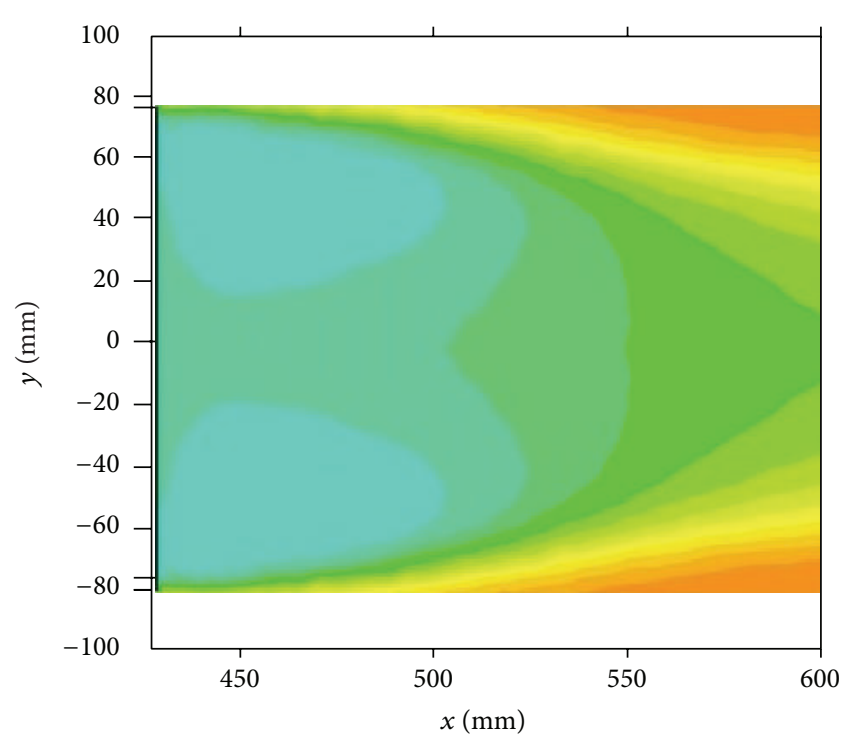

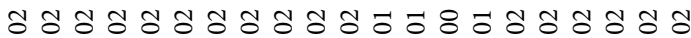
$+++++++++++++++++++++$

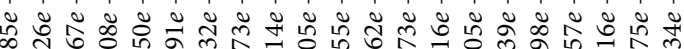
๑ సै

FIGURE 24: Total pressure in horizontal plane for basic SUV model.

and around 0.35 times the free stream velocity for the add-on model at $x=500 \mathrm{~mm}$. For the basic model, the recirculation occurs from $x=450 \mathrm{~mm}$ to $600 \mathrm{~mm}$ and for the add-on model, recirculation occurs from $x=450$ to $550 \mathrm{~mm}$. 

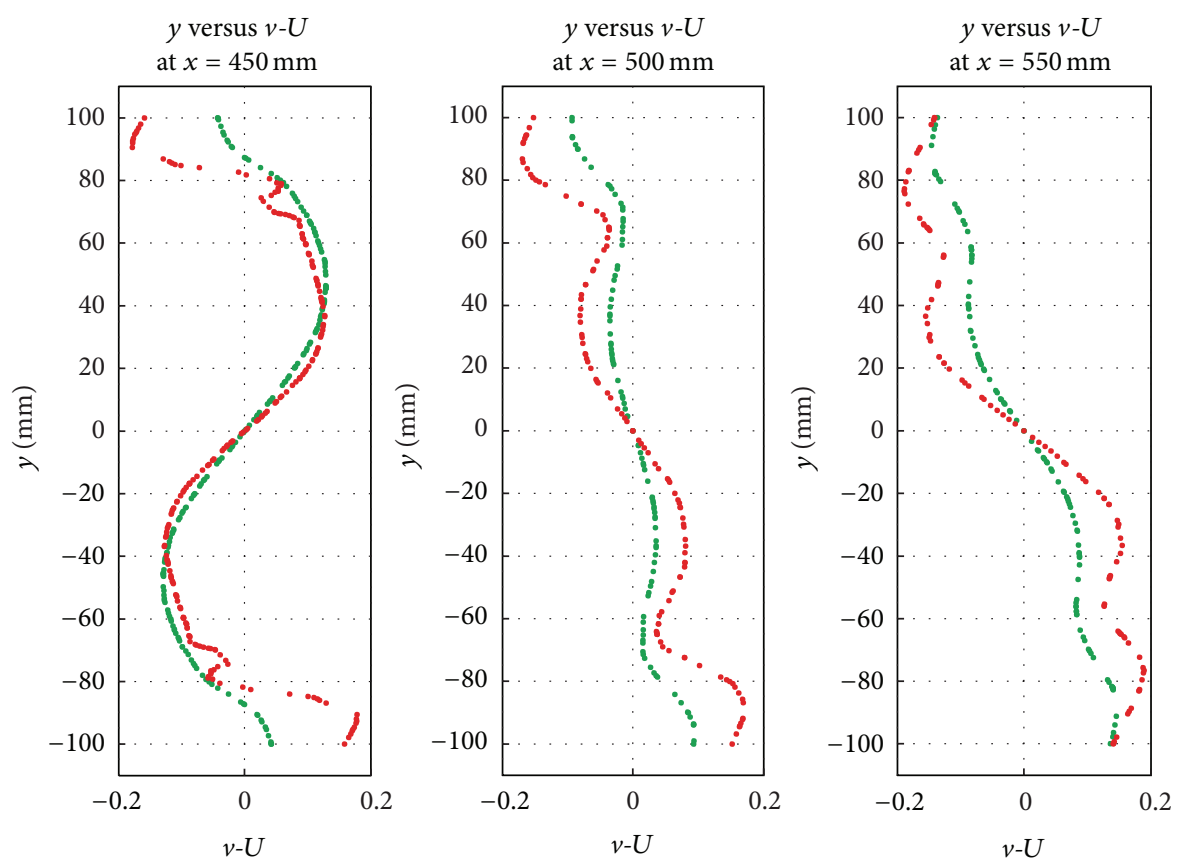

- Basic model
- Add-on model

- Basic model

- Basic model

- Add-on model
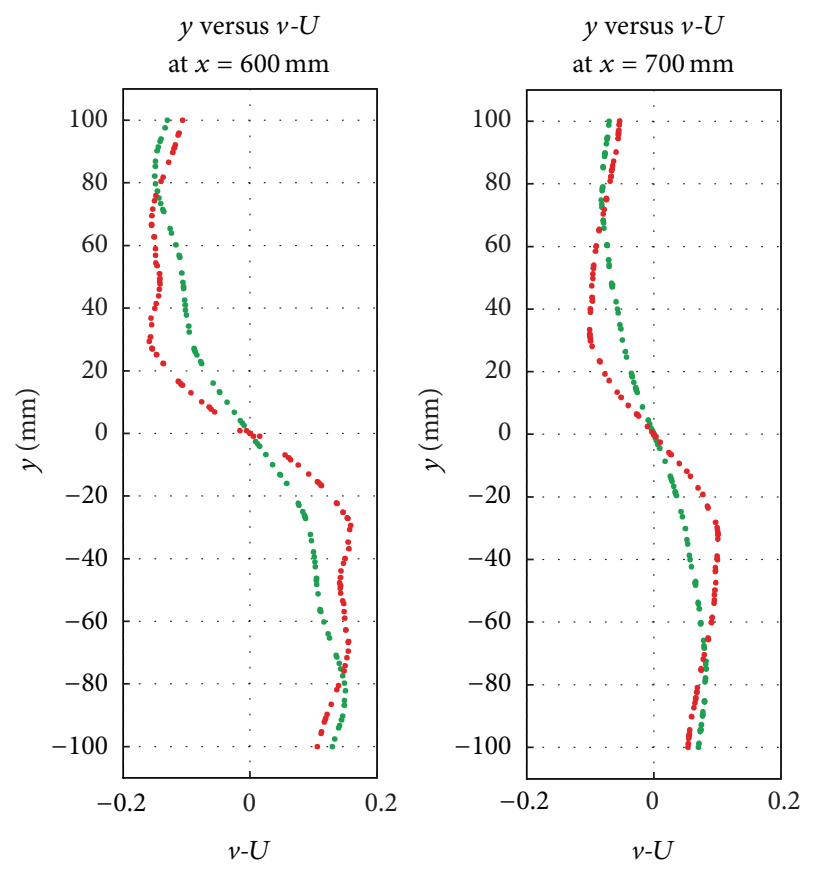

- Basic model

- Basic model

- Add-on model

- Add-on model

FIGURE 25: Mean velocity profile comparison in horizontal plane, $y$ versus $v$ - $U$.

7.3. Symmetry Plane. Symmetry plane divides the vehicle into two symmetrical parts and lies along the $Z X$ plane in the coordinate system. Velocity vectors in the symmetry plane for the wake region are shown in Figures 27 and 28 for both the add-on and the basic models. Formation of recirculation vortices is clearly visible in the figure and its length varies in both cases. For the basic model, $L$ (length of recirculation) = $150 \mathrm{~mm}$ (1.20 times the height of the base) and $L$ for the addon model $=140 \mathrm{~mm}$ (1.12 times the height of the base). By the steady wake drag model [3], the lower the length of the recirculation region is, the higher the pressure in the base region is and hence the lower the total drag on the SUV is. 

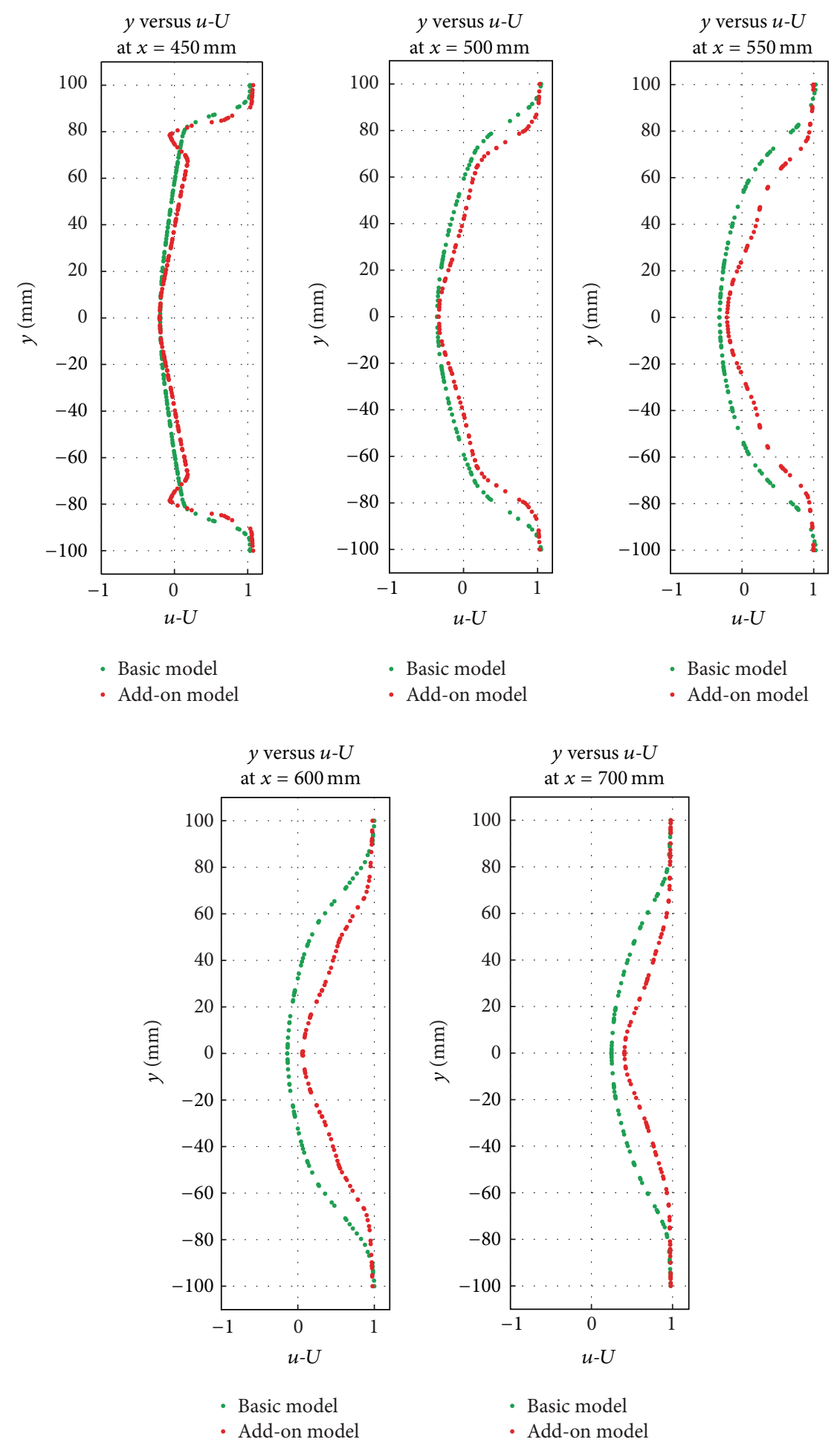

FIGURE 26: Mean velocity profile comparison in horizontal plane, $y$ versus $u$ - $U$.

Velocity contours in the symmetry plane for both cases are shown in Figures 29 and 30. Wake region extends up to a length of around $750 \mathrm{~mm}$ for the basic model. For the addon model, the wake region extends up to $670 \mathrm{~mm}$. The lower is the length of the wake region, the lower is the drag force on the SUV. Total pressure values in the symmetry plane are shown in Figures 31 and 32 for both cases. Values of total pressure are compared in Table 2 for different values of $x$ ( $\mathrm{mm}$ ) and $z=60 \mathrm{~mm}$.

Figure 33 shows the mean velocity profiles in the symmetry plane for basic and add-on models for the vertical velocity component. A strong upsweep can be seen for the add-on 


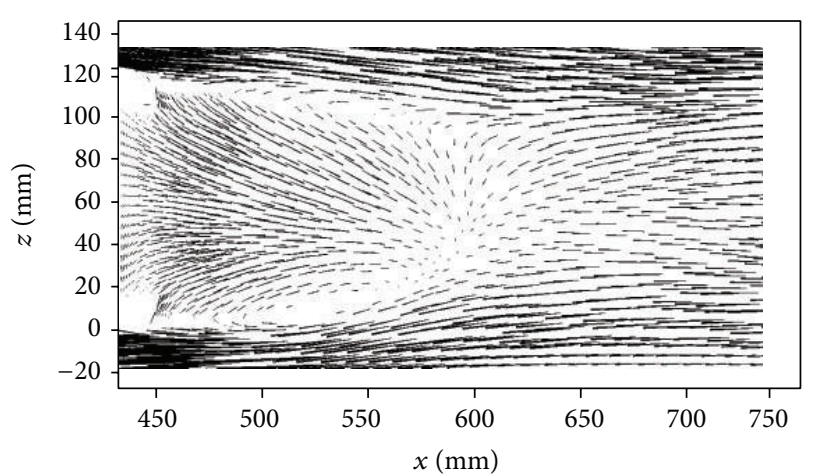

FIGURE 27: Velocity vectors for add-on model in symmtry plane.

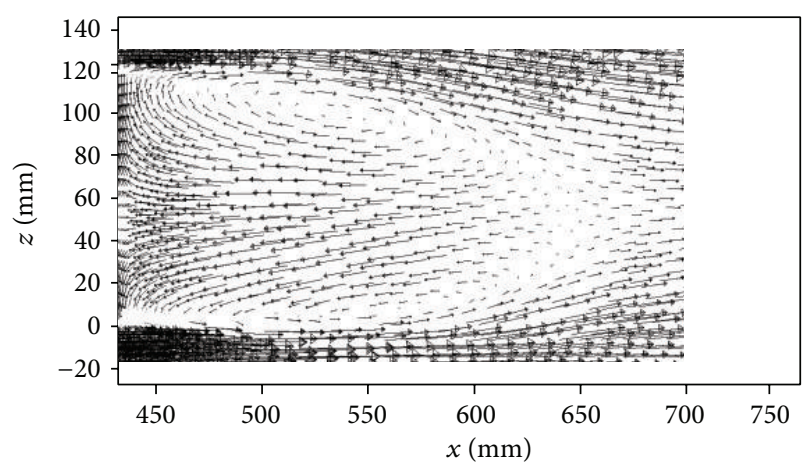

Figure 28: Velocity vectors for basic model in symmtry plane.

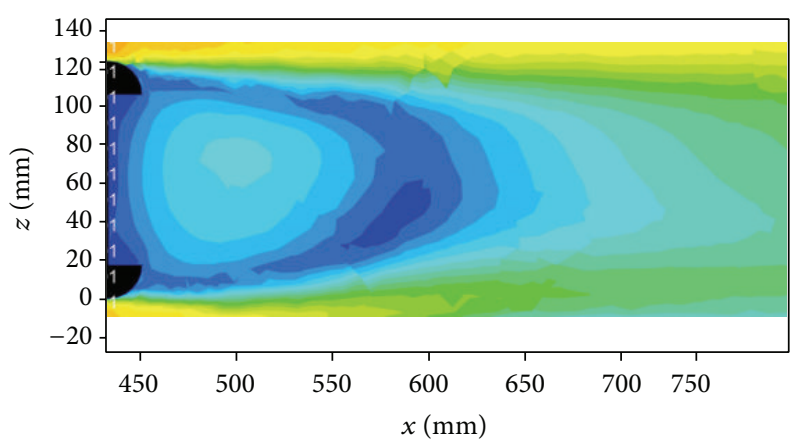

FIGURE 29: Velocity contour for add-on SUV model in symmetry plane.

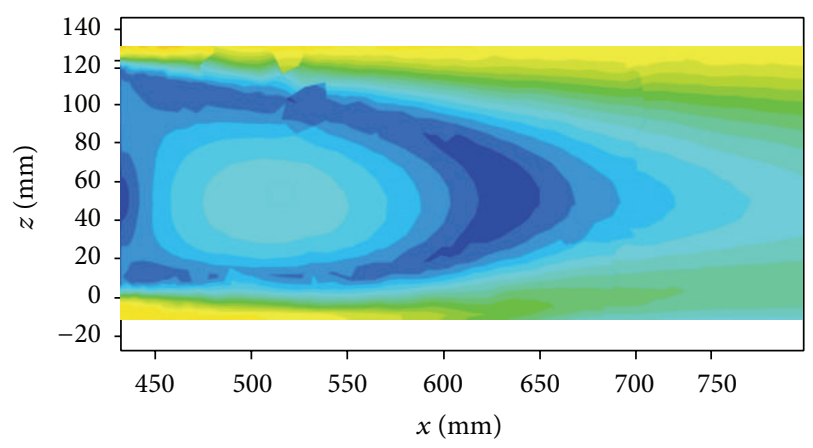

FIGURE 30: Velocity contour for basic model in symmetry plane.
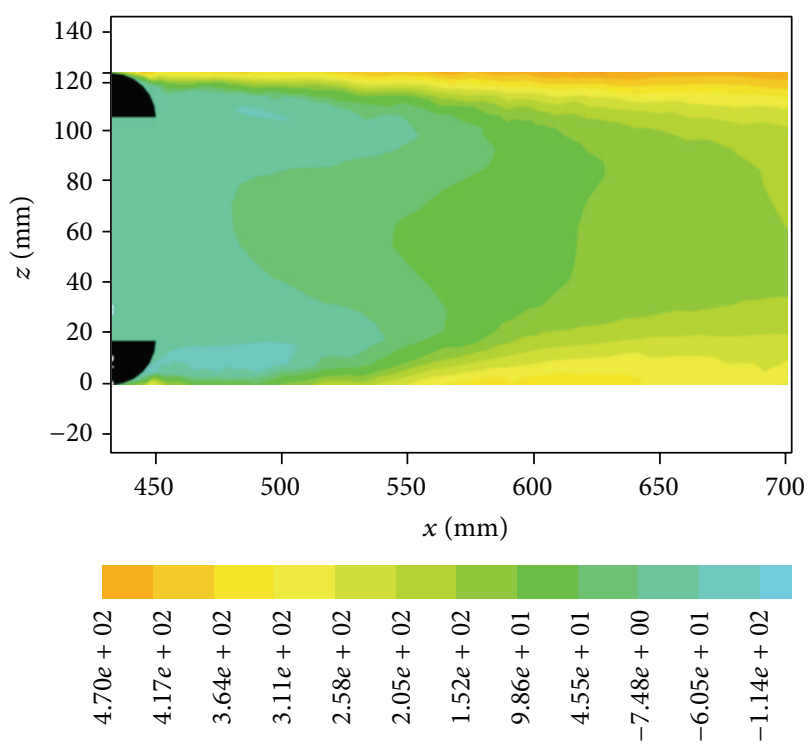

FIGURE 31: Total pressure for add-on model SUV in symmetry plane.

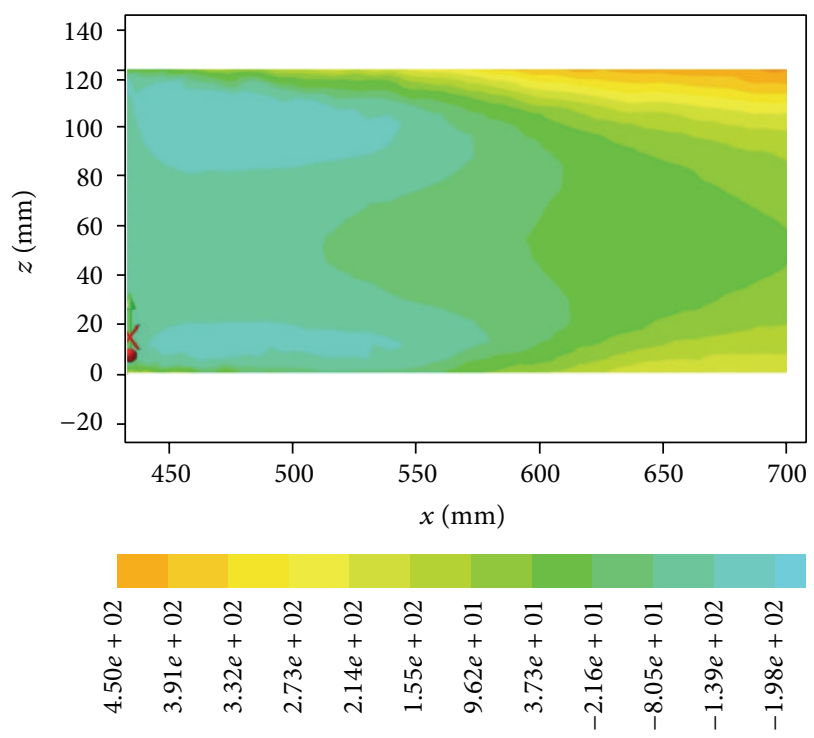

FIGURE 32: Total pressure for basic model SUV in symmetry plane.

TABLE 2: Comparison of total pressure values in symmetry plane.

\begin{tabular}{|c|c|c|}
\hline $\begin{array}{l}X \\
(\mathrm{~mm}) \\
\end{array}$ & $\begin{array}{c}\text { Basic model pressure } \\
(\mathrm{Pa})\end{array}$ & $\begin{array}{l}\text { Add-on model pressure } \\
(\mathrm{Pa})\end{array}$ \\
\hline 500 & -139 & -60.5 \\
\hline 550 & 37.3 & 98.5 \\
\hline 600 & 96.2 & 98.5 \\
\hline 650 & 96.2 & 152 \\
\hline
\end{tabular}

model from $x=500$ to $x=650 \mathrm{~mm}$. The highest value of the vertical velocity component comes out to be 0.18 times the free stream velocity for $x=500 \mathrm{~mm}$ in the add-on model and around 0.15 times the free stream velocity at $x=450 \mathrm{~mm}$ for the basic model. The Coanda effect can be clearly seen on the 


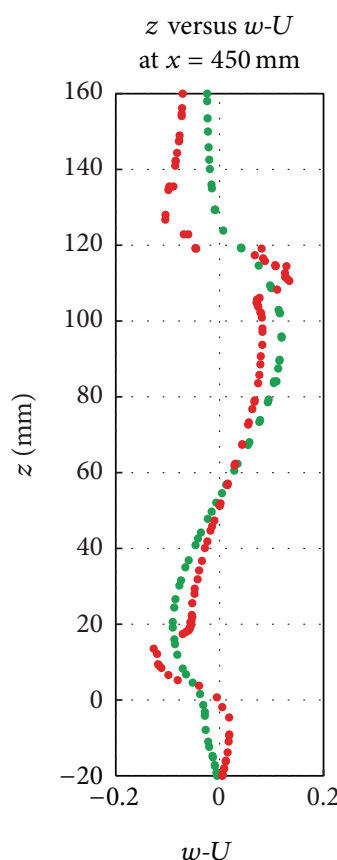

- Basic model

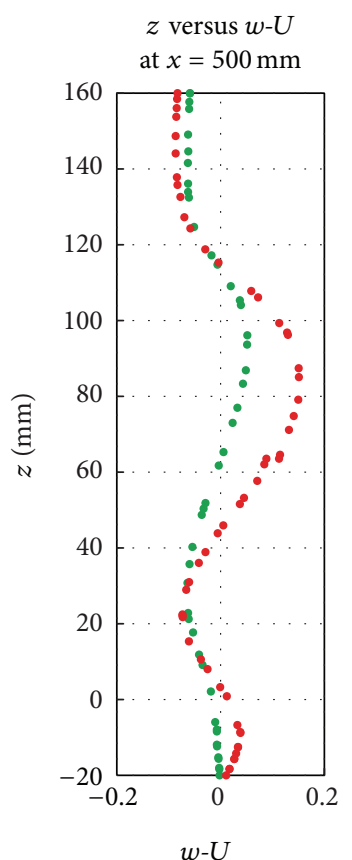

- Basic model

- Add-on model

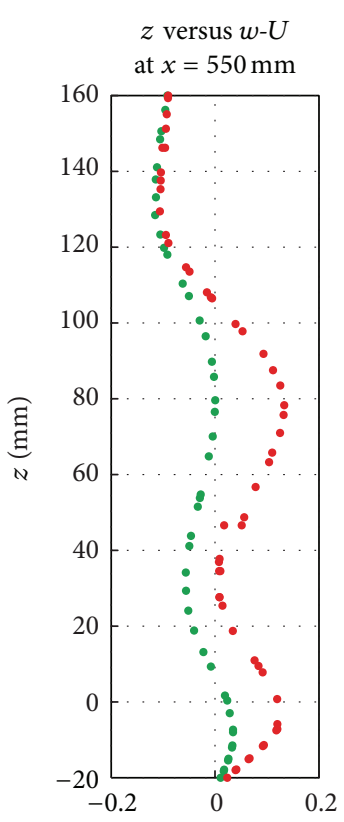

$w-U$

- Basic model

- Add-on model

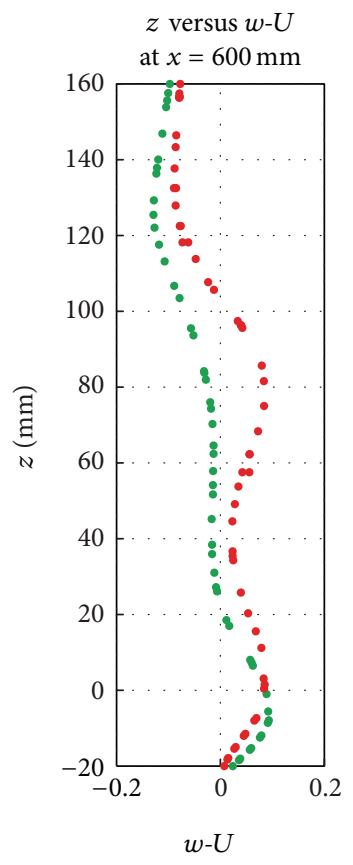

- Basic model

- Add-on model

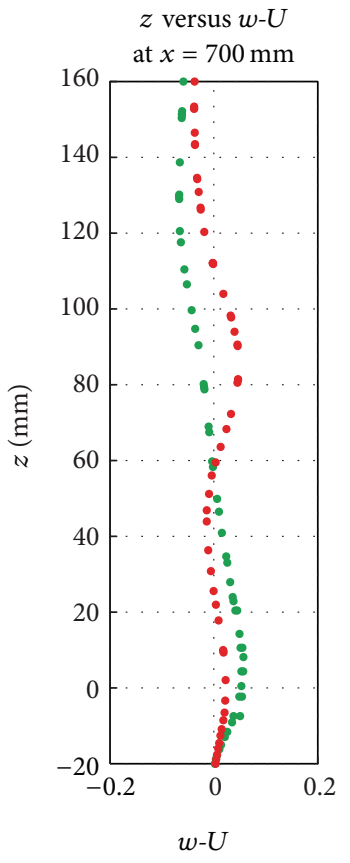

- Basic model

- Add-on model

FIGURE 33: Mean velocity profile comparison in symmetry plane, $z$ versus $w$ - $U$.

graph for $x=450 \mathrm{~mm}$ in the add-on model due to a sudden rise and fall of the velocity component at $z=110 \mathrm{~mm}$.

Figure 34 shows the mean velocity profiles in the symmetry plane for both the basic and the add-on models for horizontal velocity component. Maximum recirculation velocity is 0.3 times the free stream velocity for both models over the distances of $600 \mathrm{~mm}$; recirculation of air in the wake region is diminished for the add-on model, while a little recirculation is still present for the basic model. In the underbody region ( $z=-20$ to $60 \mathrm{~mm}$ ) the velocity component is higher for the add-on model as compared to the basic model. 

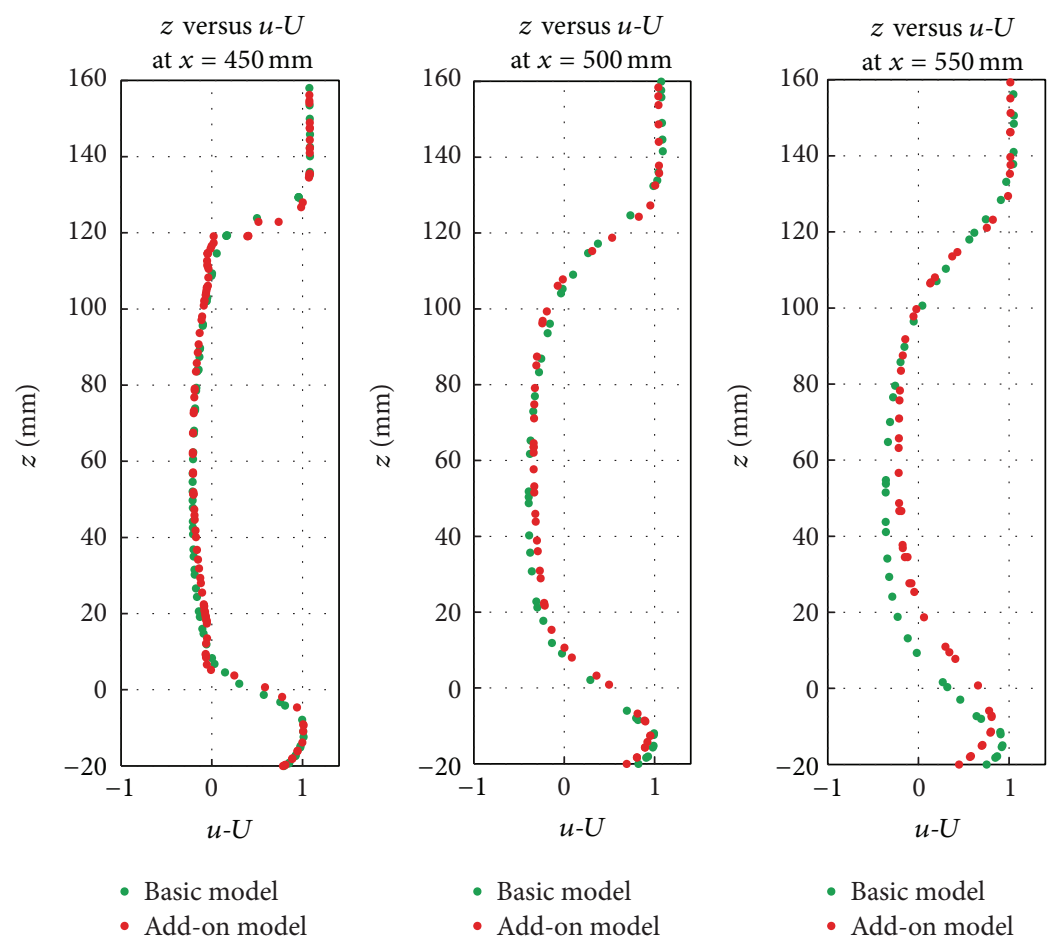

- Add-on model

$$
\text { - Add-on model }
$$

- Basic model

- Add-on model
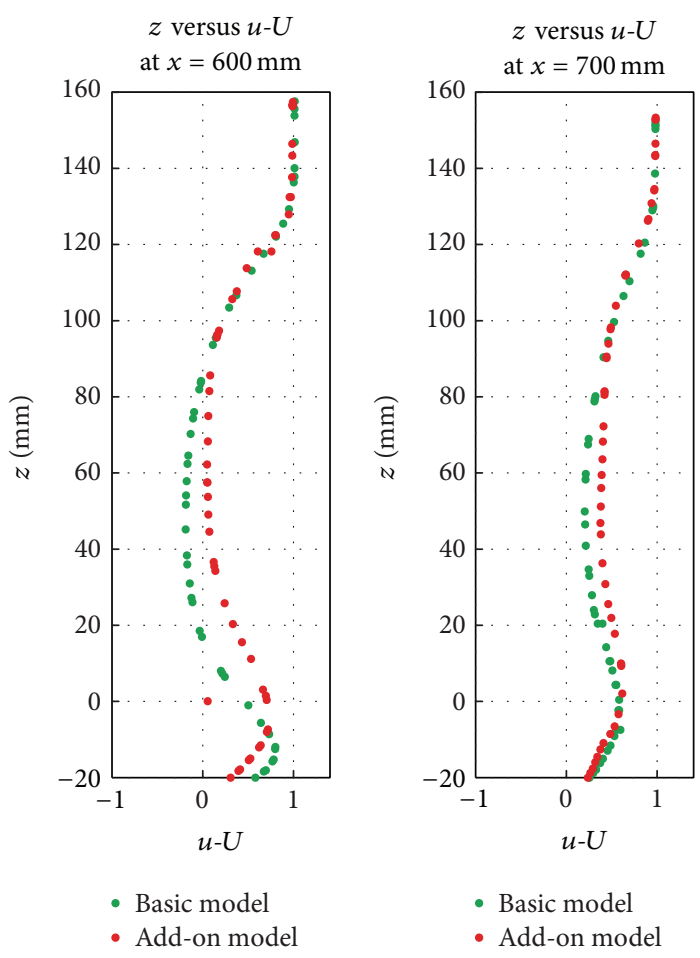

FIGURE 34: Mean velocity profile comparison in symmetry plane, $z$ versus $u-U$.

7.4. Rear Vertical Plane. Coefficients of pressure values are compared in the base plane of the SUV and are shown in Figures 35 and 36 . For the maximum portion of the area, the $\mathrm{Cp}$ values range according to the following values:

basic model: -0.288 to -0.158 ;

add-on model: -0.192 to -0.0579 .
$\mathrm{Cp}$ values and the pressure are higher for the add-on model as compared to the basic model at the base region and hence overall drag is reduced over the SUV.

Similarly, Figures 37 and 38 show the total pressure contours in the base region for both cases. Over the maximum area, the total pressure values are constant and are given below: 


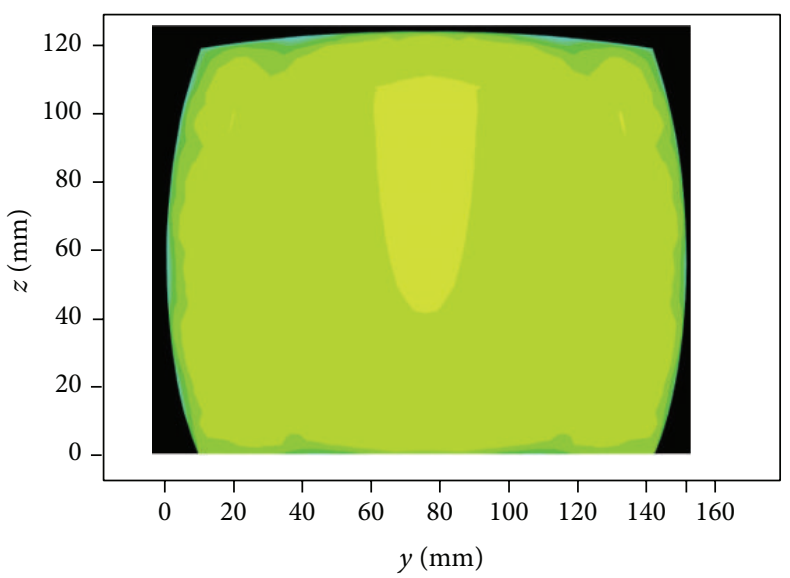

$\begin{array}{cccccccccccccccccc}+ & 1 & 1 & 1 & 1 & 1 & 1 & 1 & 1 & 1 & 1 & 1 & 1 & + & + & + & 0 & 0\end{array}$

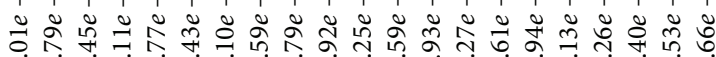

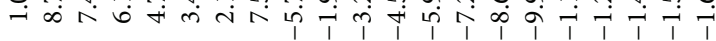

FIGURE 35: Cp values on the base of add-on model SUV.

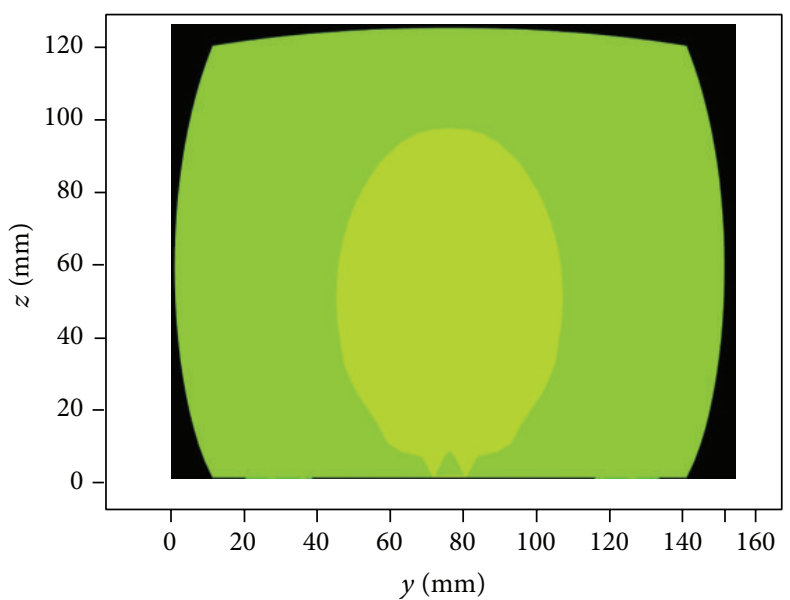

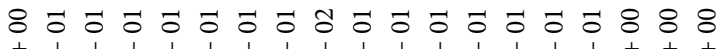
ठั

FIGURE 36: Cp values on the base of basic model SUV.

basic model: -21.6 to $37.3 \mathrm{~Pa}$;

add-on model: -7.48 to $45.5 \mathrm{~Pa}$.

Total pressure values are higher for the add-on model case over most of the base region of the SUV. Hence, the higher is the pressure in the base region, the lower is the overall drag on the SUV.

7.5. Comparison of Force and Pressure Results. Table 3 lists the various other aerodynamic parameters which are compared for both models.

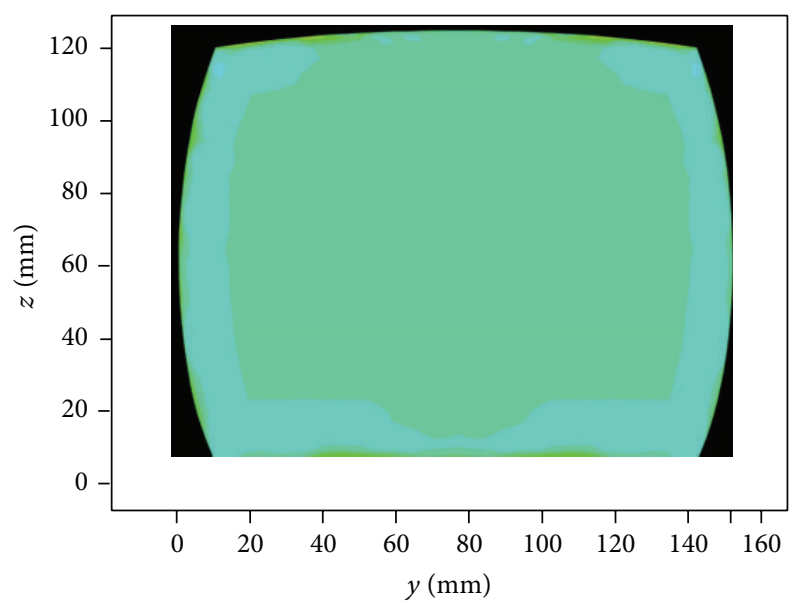

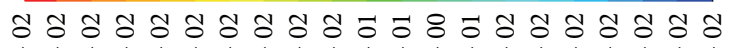
ปั ถู ம்

FIGURE 37: Total pressure values on the base of add-on model SUV.

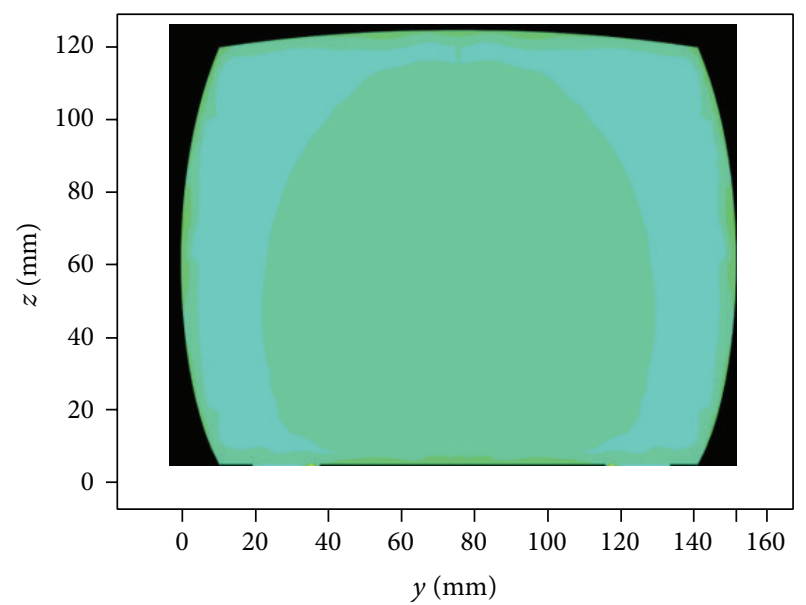

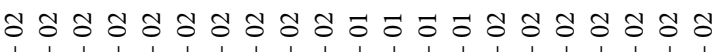
ஸั ○

FIGURE 38: Total pressure values on the base of basic model SUV.

Results clearly show that the drag forces on the addon model are lower as compared to the basic model. Also the total pressure and $\mathrm{Cp}$ on the base point are higher in magnitude for the add-on model leading to lesser drag force.

\section{Conclusion}

CFD results for a generic SUV design have been presented in this paper. An add-on inspired by the GETS model is added to the generic SUV and CFD analysis is performed on it. Comparison of various aerodynamic parameters is 
TABLE 3: Comparison of aerodynamic parameters for add-on model and basic model SUV.

\begin{tabular}{lccc}
\hline S. number & $\begin{array}{c}\text { Aerodynamic } \\
\text { parameter }\end{array}$ & $\begin{array}{c}\text { Basic SUV } \\
\text { model }\end{array}$ & $\begin{array}{c}\text { Add-on SUV } \\
\text { model }\end{array}$ \\
\hline 1 & Form drag & $1.8696 \mathrm{~N}$ & $1.6824 \mathrm{~N}$ \\
2 & $\begin{array}{c}\text { Form drag } \\
\text { coefficient }\end{array}$ & 0.3140 & 0.2824 \\
3 & $\begin{array}{c}\text { Total force } \\
\text { Total pressure } \\
\text { on base point } \\
\text { Cp on base } \\
\text { point }\end{array}$ & $-47 \mathrm{~Pa}$ & $-19 \mathrm{~Pa}$ \\
5 & $\begin{array}{c}\text { Total coefficient } \\
\text { of drag }\end{array}$ & 0.3569 & -0.035 \\
6 & $\begin{array}{c}\text { Total coefficient } \\
\text { of lift }\end{array}$ & $2.74 * 10^{-2}$ & $4.79 * 10^{-2}$ \\
\hline
\end{tabular}

done to establish the effectiveness of the add-on. The various conclusions from this study can be summarized in the following points.

(1) Coefficient of drag for the add-on SUV has reduced to 0.3283 , giving a total reduction of $8.013 \%$.

(2) Cp plots for both cases are similar, except at the end portion of the curve due to the Coanda effect, and also the flow is attached to the add-on on the upper rear region of the SUV which leads to pressurization of the wake region.

(3) Total pressure on the base point of the SUV model has increased on addition of the add-on leading to higher pressure in the wake region and hence lower overall drag, according to the steady wake drag theory. Total drag force on the SUV model has reduced from $2.1251 \mathrm{~N}$ to $1.9557 \mathrm{~N}$.

(4) Length of the recirculation region reduces from 1.2 to 1.12 times the width of the SUV in the symmetry plane which is a characteristic of the flow region in the wake.

(5) The wake region for the SUV has reduced in length from $750 \mathrm{~mm}$ to $670 \mathrm{~mm}$. The shorter is the length of the recirculation region, the lesser is the drag force on the SUV.

(6) The width of the wake region has also reduced. Location of the recirculation vortices has changed from $\pm 55 \mathrm{~mm}$ to $\pm 35 \mathrm{~mm}$ in the centre horizontal plane.

(7) Total pressure and the Cp values on the base point of the SUV increased which explains the low overall drag value on the add-on model. Hence, overall reduction on the drag force on the SUV leads to increase in the fuel efficiency of the SUV vehicle.

\section{Future Work}

(1) Till now, the computational analysis of the problem has been done using the CFD and significant results showing the improvements in the aerodynamics of the SUV have been presented. So, experimental analysis of this model is recommended. Comparison of the experimental and the CFD results could be done to establish the effectiveness of the add-on on the generic SUV.

(2) Other add-on devices like base bleed can be used on the SUV to reenergize the wake region and reduce the drag on the SUV.

(3) Vortex strake devices (VSD) have been successfully tested on trucks and trailers to energize the flow behind the trailer. VSD can be used on SUVs for producing the same effect and hence reduce the drag force.

(4) Active flow control (AFC) can be implemented on the SUV design to further reduce the drag force on the SUV and increase the fuel efficiency.

\section{Conflict of Interests}

The authors declare that there is no conflict of interests regarding the publication of this paper.

\section{References}

[1] G. K. Suryanarayana, H. Pauer, and G. E. A. Meier, "Bluff-body drag reduction by passive ventilation," Experiments in Fluids, vol. 16, no. 2, pp. 73-81, 1993.

[2] J. Englar Robert, "Advanced Aerodynamic Devices to Improve the Performance, Economics, Handling and Safety of Heavy Vehicles," SAE 2001-01-2072, Georgia Tech Research Institute Aerospace Transportation \& Advanced Systems Lab, 2001.

[3] P. M. van Leeuwen, Computational analysis of base drag reduction using active flow control [Master of Science in Aerospace Engineering], Delft University of Technology, 2009.

[4] A. Lamond, J. J. Kennedy, and M. T. Stickland, "An investigation into unsteady base bleed for drag reduction in bluff twobox SUV's," in Proceedings of the 4th European Automotive Simulation Conference (EASC '09), Munich, Germany, 2009.

[5] Y. A. Sevilla, Drag reduction of sport utility vehicle using vortex generators [M.S. thesis], California State University, Sacramento, Calif, USA, 2012.

[6] P. N. Krishnani, CFD study of drag reduction of a generic sport utility vehicle [Master of Science in Mechanical Engineering], California State University, Sacramento, Calif, USA, 2009.

[7] E. M. Wahba, H. Al-Marzooqi, M. Shaath, M. Shahin, and T. ElDhmashawy, "Aerodynamic drag reduction for ground vehicles using lateral guide vanes," CFD Letters, vol. 4, no. 2, pp. 68-79, 2012.

[8] K. Bahram, S. Zhang, C. Koromilas et al., "Experimental and computational study of unsteady wake flow behind a bluff body with a drag reduction device," SAE 2001-01B-207, 2001.

[9] A. M. Al-Garni, L. P. Bernal, and B. Khalighi, "Experimental investigation of the flow around a generic SUV," SAE 2004-010228, 2004.

[10] M. H. Coanda, "Propelling device," French Patent no. 796843, 1936. 
[11] Fluent Deutschland, Best Practice Guidelines for Handling Automotive External Aerodynamics with FLUENT, Version 1.2, Fluent Deutschland, Darmstadt, Germany, 2005.

[12] T. Gustavsson and T. Melin, "Application of Vortex generators to a blunt body," Technical Report KTH, Department of Aeronautical and Vehicle Engineering, Royal Institute of Technology, Stockholm, Sweden, 2006. 

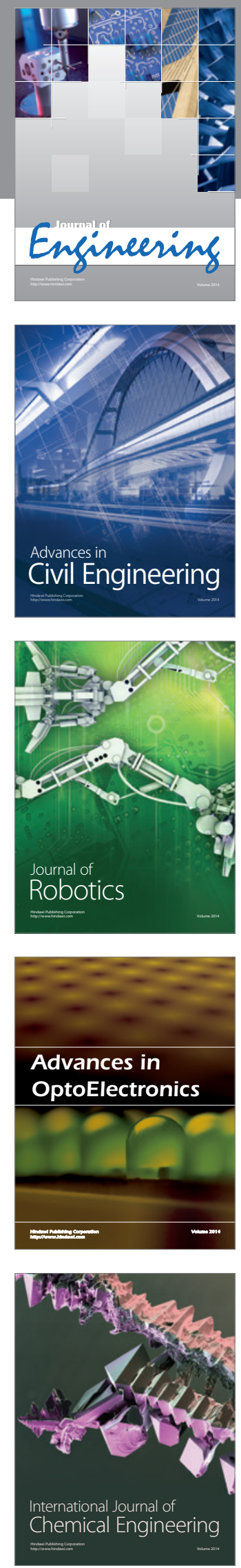

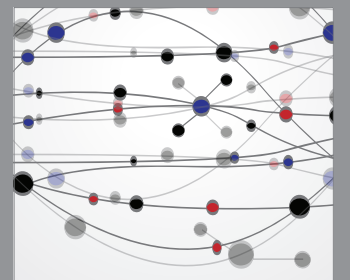

The Scientific World Journal
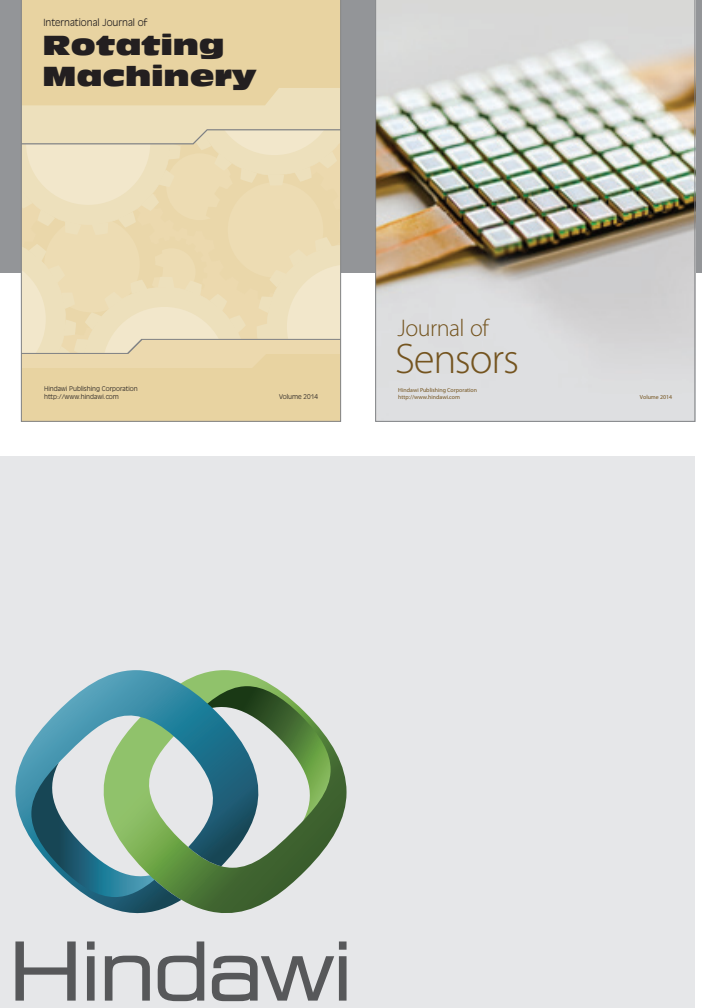

Submit your manuscripts at http://www.hindawi.com
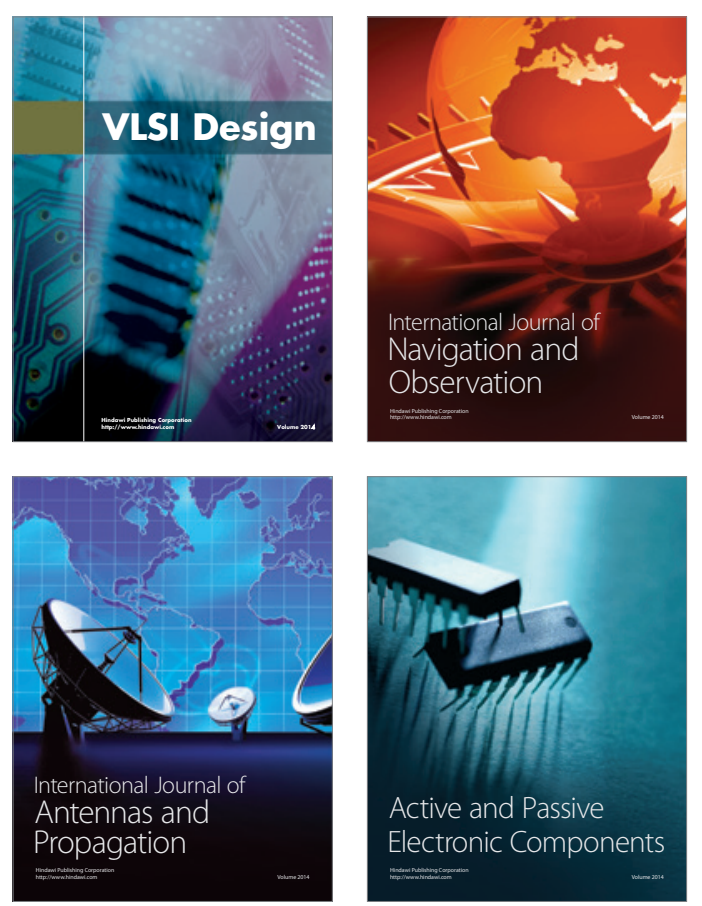
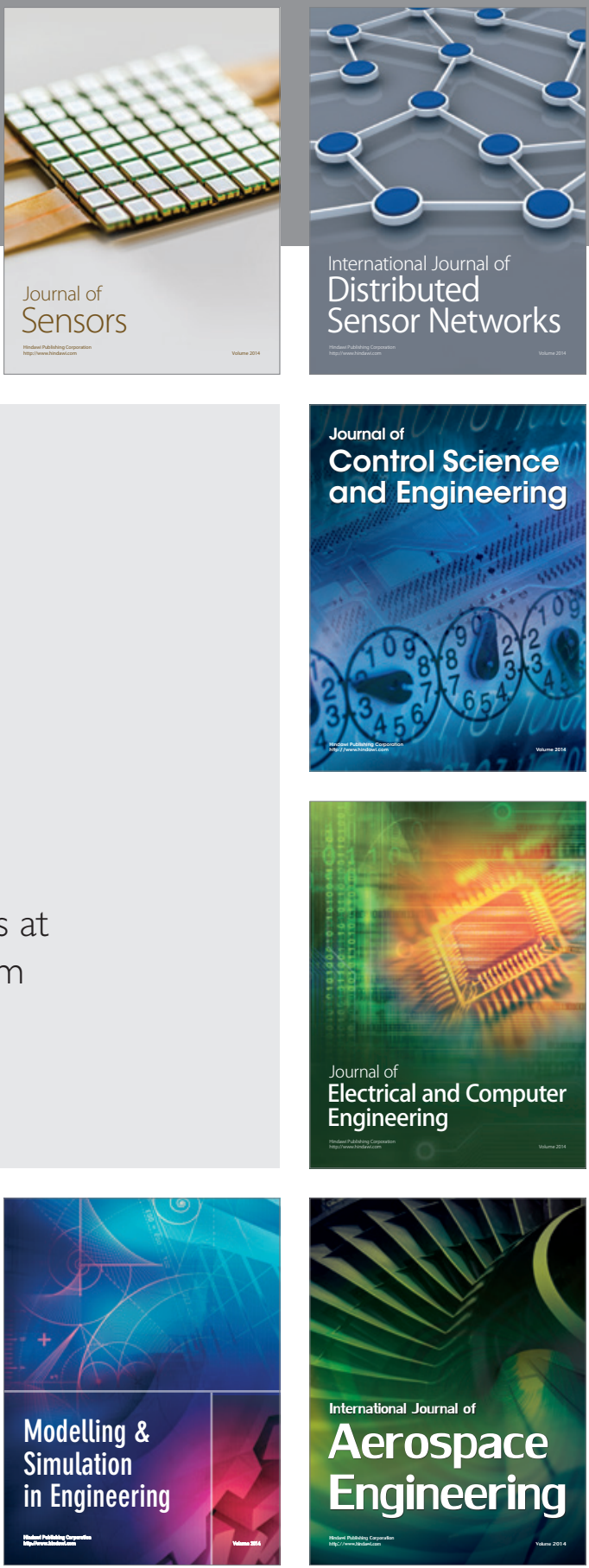

Journal of

Control Science

and Engineering
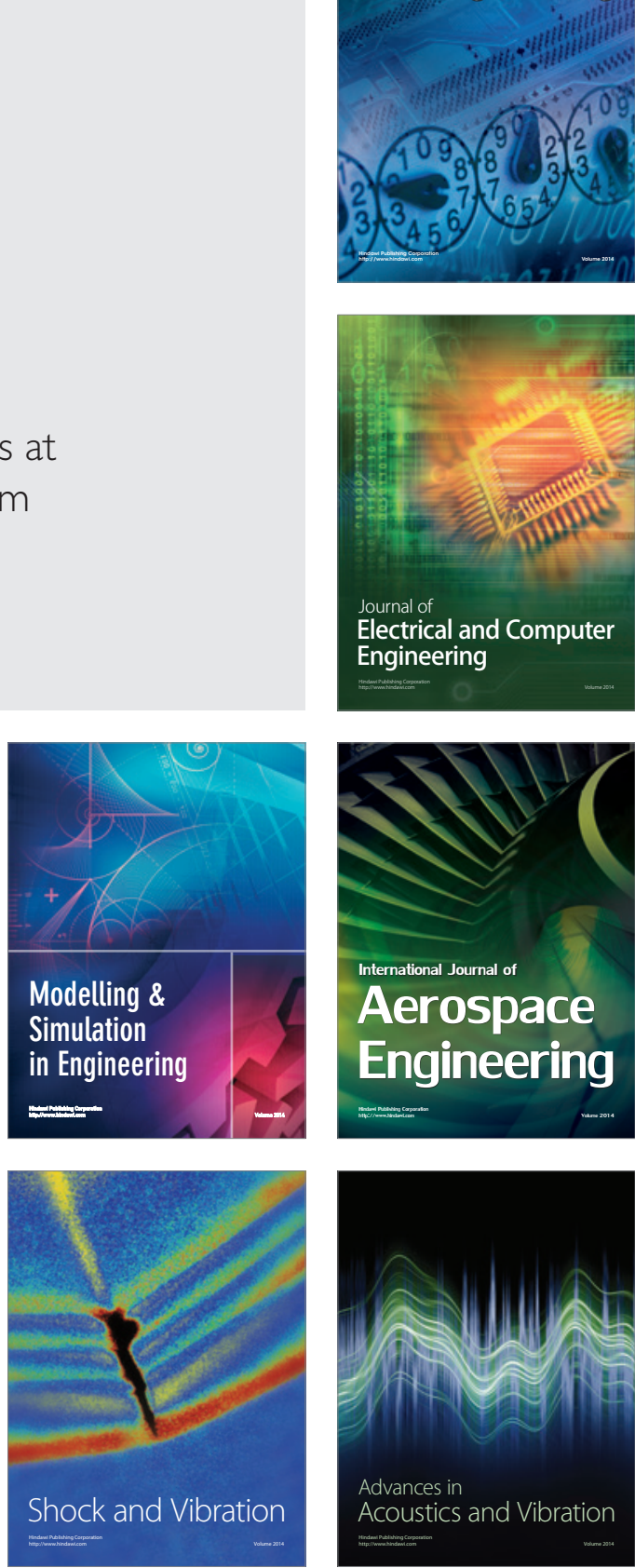